\title{
Bilevel Programming for Traffic Signal Coordinated Control considering Pedestrian Crossing
}

\author{
Dawei Li $\mathbb{D}^{1,2}$ Yuchen Song $\mathbb{D}^{3},{ }^{3}$ and Qiong Chen ${ }^{4}$ \\ ${ }^{1}$ Jiangsu Key Laboratory of Urban ITS, Southeast University, Sipailou 2, Xuanwu District, Nanjing 210096, China \\ ${ }^{2}$ Jiangsu Province Collaborative Innovation Center of Modern Urban Traffic, School of Transportation, Southeast University, \\ Sipailou 2, Xuanwu District, Nanjing 210096, China \\ ${ }^{3}$ Jiangsu Key Laboratory of Urban ITS, School of Transportation, Southeast University, Sipailou 2, Xuanwu District, \\ Nanjing 210096, China \\ ${ }^{4}$ School of Architecture, Southeast University, Sipailou 2, Xuanwu District, Nanjing 210096, China
}

Correspondence should be addressed to Yuchen Song; 213152887@seu.edu.cn

Received 16 August 2019; Accepted 4 December 2019; Published 13 February 2020

Guest Editor: Xiaofeng Chen

Copyright (c) 2020 Dawei Li et al. This is an open access article distributed under the Creative Commons Attribution License, which permits unrestricted use, distribution, and reproduction in any medium, provided the original work is properly cited.

\begin{abstract}
With the rapid development of the subway, more and more people choose it as the main method of transportation. However, practically, the large number of pedestrians near some large metro stations can also correspondingly affect the traffic of motor vehicles on the roads adjacent to the stations. In this study, coordinated control of the traffic signal which considers the pedestrian crossing delay is studied based on this background. Firstly, the model of progression band in adjacent intersections is analyzed comprehensively, and the calculation formulas of progression bandwidth and the delay of vehicles which are from the progression of traffic flow under different conditions are given. Secondly, five different models of pedestrian delay are analyzed. Under different conditions of motor vehicle and pedestrian traffic flow, the Vissim fitting and proofreading are carried out and the optimal models under different conditions are obtained. Finally, the bilevel programming problem which fuses the above two models is determined; by coding an algorithm, it can be resolved. Furthermore, taking eight signalized intersections from Jiming Temple to Daxinggong along Nanjing Metro Line 3 as the actual background, the calculation and optimization of coordinated control are carried out. It is found that at the expense of the traffic efficiency of large intersections to a certain extent, a wider progression band can be formulated on the roads between them, and pedestrian delays can be reduced in general.
\end{abstract}

\section{Introduction}

Taking Nanjing as an example, in recent years, the growth of urban motor vehicles has been obvious. The main artery roads of many cities are in near-saturated state all the year round. This has brought great negative impacts on our traveling, which not only affects the efficiency of work, but also causes people emotional dissatisfaction, so it is an important mission of our traffic workers to improve traffic capacity and maximize traffic efficiency within a limited road space $[1,2]$.

Through the coordinated control of traffic signals, we can meet people's traffic needs to the maximum extent while ensuring safety [3-5]. The function of an isolated intersection is mainly determined by its control parameters, and of course by other intersections. However, due to the complexity of modern transportation systems, coordinated control of traffic signals has become one of the important traffic management strategies in cities [6,7]. Mapping to reality is even more unimpeded traffic flow, fewer traffic accidents, and more stable driving speed [8].

Therefore, it is particularly important to coordinate the signals at the intersections around the subway stations where congestion is increasing and traffic demand is in expansion [9]. The project will use microsimulation as a research tool to analyze the main factors that need to be considered in the coordinated control of traffic signals. The time-space conflict between the vehicle and its influence on the coordinated 
control of the signals proposes a traffic signal control method that is conducive to subway passengers' travel $[10,11]$.

Here is the literature review of signal coordinated control. In terms of coordinated control of signals, as early as the 1970s, there was a MAXBAND model for coordinated control of artery roads [12]. The concept of a progression band was proposed. The two-way progression bandwidth was taken as the objective function, and the maximum objective function model was used, combining mixed integer linear programming. Then there is the MULTIBAND model $[13,14]$, which is based on different traffic requirements on different road sections, and the corresponding bandwidth is calculated to get the best coordination effect. For the regional coordinated control, an off-line optimized network signal timing of TRANSYT proposed by the British Road Traffic Research Institute has been developed to comprehensively consider the comprehensive model of cruise time, bus modeling, peak delay calculation, and fuel consumption estimation [15], as well as inductive regional traffic coordination control systems SCATS, SCOOT, and others, which can effectively improve the traffic conditions of unsaturated and supersaturated road networks [16].

After combining the bus priority strategy, Vasudevan [17], under the framework of bus priority coordination control, with the minimum green time as the constraint and the minimum per capita delay under coordinated control as the optimization goal, established the optimization of trunk coordination control based on bus priority model. Many advanced signal control systems take into account different priority transit strategies and are added to the algorithm to better and more efficiently adapt to multimode control needs. Conversely, some systems are designed to reduce per capita delays, such as the reduction of motor vehicle delays. Some studies $[18,19]$ have added traffic priority based on traffic rules, and, for Lo [20], a series of motor vehicles are selected in advance to implement their priority control methods. In addition, some systems weigh the factors such as pedestrian delays, motor vehicle delays, bus delays [21], or by reducing the transit time of buses and the waiting time of pedestrians on downstream stations and other priority strategies for other transportation systems [22].

Recently, signal coordination control has increasingly favored the use of vehicle-coordinated data, and they have attempted to combine all of the above factors, including weighing the delays of various passers-by, taking into account various priority strategies. In the system operation, real-time traffic information needs to be obtained. Tan realized vehicle information interaction through AVL (Automated Vehicle Location) or APC (Automated Passenger Counter) [23]. However, such systems are limited to two connected main road intersections (not more), and there are more problems with multiple bus lines and multiple priority control strategies [11]. There are still researches using machine learning methods to deal with the problems within the intersection between vehicles and pedestrians [24-26]; however, the results are limited and unexplainable for traffic design and control [27, 28].
Although there are some researches focusing on the modeling of pedestrian crossing behavior [29-31], to the best of our knowledge, it is rare to combine models of coordinating signal control and pedestrian delay. So, we consider the problems of pedestrian crossing with signal control. In this paper, the situation of green light time and relative offset between two adjacent intersections is analyzed comprehensively. Then, according to the average travel time of the motor vehicle, the green wave feasibility analysis is carried out to calculate the corresponding progression bandwidth and the delay of the motor vehicle flowing under the green wave (not considering the delay of the nongreen wave motor vehicle). Finally, the complete intersection green wave analysis is obtained. Then, the model of pedestrian delay is analyzed. By collecting a large amount of data, the model of a different motor vehicle and pedestrian flow delays is obtained. Then, the vehicle flow and pedestrian flow are simulated by Vissim to test the simulation of each model under different conditions. The degree of integration is obtained to generate a more accurate calculation model of pedestrian delay. Secondly, the algorithm is written in Python, and the above two models are merged together and applied to the actual intersection coordination model, and the optimal coordination scheme is obtained by inputting into the algorithm. Finally, the Vissim is used to simulate the actual situation to judge the rationality of the established model and the edited algorithm and draw conclusions.

The remaining part of this paper is as follows: in section 2, we put forward models of progression bandwidth under different situations of cycle length, green time, and offset. Section 3 illustrates the process of establishing models of pedestrian delay. Section 4 proposes the bilevel programming fusing the aforementioned two models and an algorithm to solve it. Furthermore, it also provides a case study to test the reasonableness of them. Finally, we conclude in Section 5 .

\section{Model of Progression Bandwidth}

Due to the high land utilization rate in large cities, the distance between adjacent intersections is very close. Therefore, it is necessary to coordinate the signal scheme to maximize the traffic efficiency of the vehicle. This is called artery traffic coordination control. The so-called green wave means that the vehicle (vehicle flow) continuously passes through a certain main road and encounters less red light as much as possible.

There are many parameters in the signal coordination control, including signal period, green signal ratio, offset, and so on. The main idea of building a model is obtained [32], which is modeled by drawing a spatiotemporal map. This paper mainly studies the coordinated control of two adjacent intersections and calculates the delay and progression bandwidth generated by the corresponding green wave traffic by considering the situation of the vehicle queuing at the second intersection. This paper is based on and beyond the content of the former, and at the same time, corrects some errors. Through the comparison of the red and green light phase difference between the two intersections 
and the first intersection lipstick and green light time, the complete signal set of adjacent intersections is summarized. Then, the subclass is divided by the average transit time of the motor vehicle, and then the coordination parameters of each subclass are analyzed in turn, and finally, the complete model is obtained.

Because the coordinated control is affected by multiple factors, the vehicle discretization is serious, resulting in the formation of a fleet; there are a large number of vehicles entering and exiting the road, thus destroying the formation of continuous traffic. The signal lights at the intersection are complex, with more than two phases and coordination difficulties. Therefore, before establishing a model, it is necessary to make some reasonable assumptions, both not to deviate from the actual situation and to meet the conditions for establishing the model.

(1) The effects of road traffic conditions, such as weather and road geometry, are not considered.

(2) The road between intersections does not contain a large number of vehicles that are transferred in or out and does not contain a large number of important traffic points that attract people and passengers.

(3) The distance between the intersections should not exceed $600 \mathrm{~m}$.

(4) The driving speed of the vehicle does not fluctuate much, and there is no large difference in the speed between the vehicles.

(5) The intersections that need to be coordinated are controlled by two phases, the period length is the same, and only the red and green lights are considered (the yellow light is counted in the green light), and the pedestrian emptying time is not included.

(6) The coordinated sections are all unsaturated.

The symbols are defined in Table 1, and the relationship of some of them is given as follows (specific symbols in models will be given correspondingly):

$$
\begin{aligned}
\Delta_{i, i+1}^{g} & =\Delta_{i, i+1}^{r}+r_{i+1}-r_{i}, \\
r_{i} & =C-g_{i}, \\
t_{i, i+1} & = \begin{cases}\frac{d_{i, i+1}}{v}, & \frac{d_{i, i+1}}{v}<C, \\
\frac{d_{i, i+1}}{v} \bmod C, & \frac{d_{i, i+1}}{v} \geq C .\end{cases}
\end{aligned}
$$

In Figure 1, when $\Delta_{i, i+1}^{r}>r_{i}, \Delta_{i, i+1}^{g}<g_{i}$, according to the position of the last intersection corresponding to the start time of the red light at the next intersection, it is divided into three parts of four categories. The slope of red lines in Figure 2 is the basis of classification.

Category I $\left(t_{i, i+1}<\Delta_{i, i+1}^{r}-r_{i}\right.$ (Figure 1 part (1))): in Figure 2(a), at this moment, some vehicles departing
TABLE 1: Notations of model symbols and variables.

\begin{tabular}{lc}
\hline Symbol & Illustration \\
\hline$I_{i}$ & $i$-th signal \\
$C$ & Cycle length $(\mathrm{s})$ \\
$r_{i}$ & Red time at $I_{i}(\mathrm{~s})$ \\
$g_{i}$ & Green (and yellow) time at $I_{i}(\mathrm{~s})$ \\
$\Delta_{i, i+1}^{r}$ & Offset of red starting time between $I_{i}$ and $I_{i+1}(\mathrm{~s})$ \\
$\Delta_{i, i+1}^{g}$ & Offset of green starting time between $I_{i}$ and $I_{i+1}(\mathrm{~s})$ \\
$q_{i}^{r}$ & Traffic volume at $I_{i+1}$ when $I_{i}$ is red $(\mathrm{pcu} / \mathrm{s})$ \\
$q_{i}^{g}$ & Traffic volume at $I_{i+1}$ when $I_{i}$ is green $(\mathrm{pcu} / \mathrm{s})$ \\
$S_{i}$ & Saturated flow rate at $I_{i}(\mathrm{pcu} / \mathrm{s})$ \\
$t_{i, i+1}$ & Average travel time between $I_{i}$ and $I_{i+1}(\mathrm{~s})$ \\
$b_{i}$ & Progression bandwidth $(\mathrm{s})$ \\
\hline
\end{tabular}

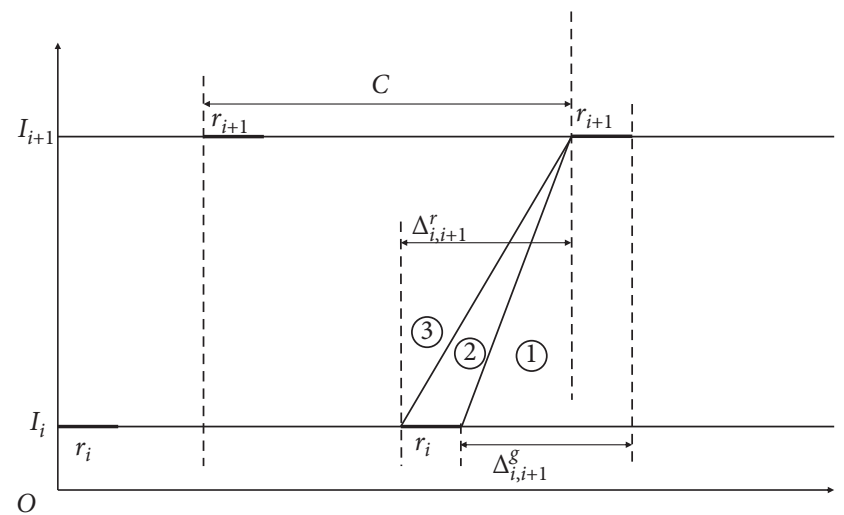

Figure 1: Classification of interlaced spatiotemporal figures according to offset.

from the green light at the intersection $i$ can pass through the $i+1$ th intersection, and some of them have to wait for congestion. $t_{2}$ denotes the time interval when vehicles meet with congestion at the specified speed, and $t_{3}$ denotes the congestion evacuation time (for the evacuation through the parking line, the same below). $D$ denotes traffic delay of vehicles from the progression band, which is represented by the shadowed part in this figure. So we can derive the following equations:

$$
\begin{aligned}
& \left\{\begin{array}{l}
t_{2}=t_{3}+r_{i+1}, \\
t_{2} q_{i}^{g}=t_{3} S_{i+1}, \\
b_{i}=g_{i}-t_{2},
\end{array}\right. \\
& b_{i}=g_{i}-\frac{r_{i+1} S_{i+1}}{S_{i+1}-q_{i}^{g},} \\
& D=\frac{r_{i+1} t_{2} q_{i}^{g}}{2}=\frac{r_{i+1} q_{i}^{g}}{2} * \frac{r_{i+1} S_{i+1}}{S_{i+1}-q_{i}^{g}} .
\end{aligned}
$$

Category II $\left(\Delta_{i, i+1}^{r}-r_{i}<t_{i, i+1}<\Delta_{i, i+1}^{r}\right.$ (Figure 1 part (2))): in Figure 2(b), the vehicles from ith intersection at red time and the link connecting $i$ th and $i+1$ th intersection generate queue. So when the progression vehicles come to 


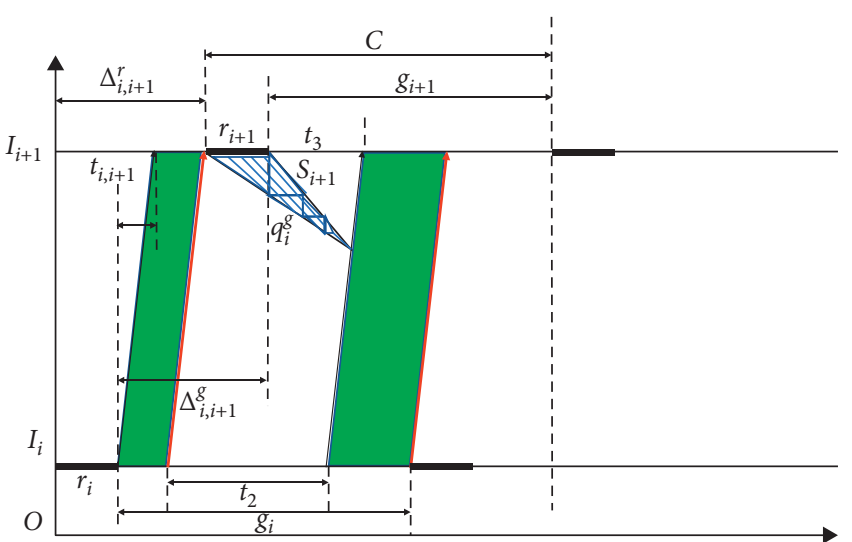

(a)

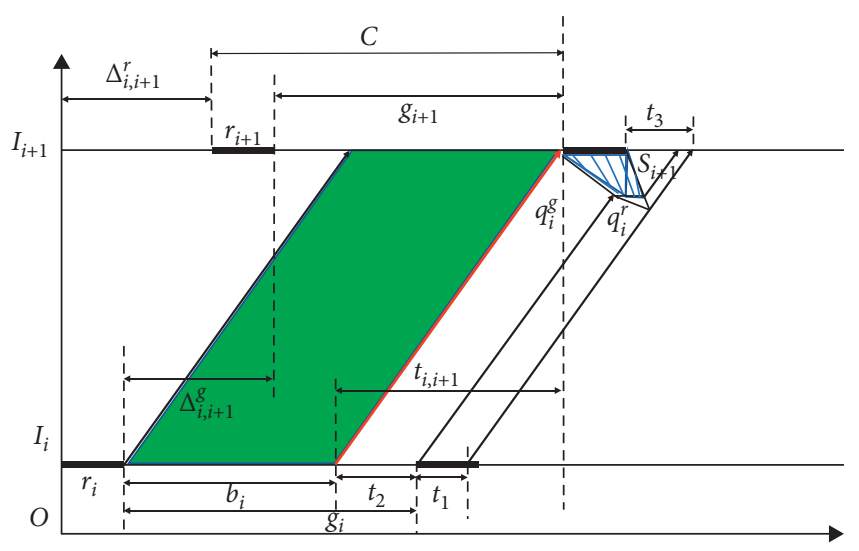

(c)

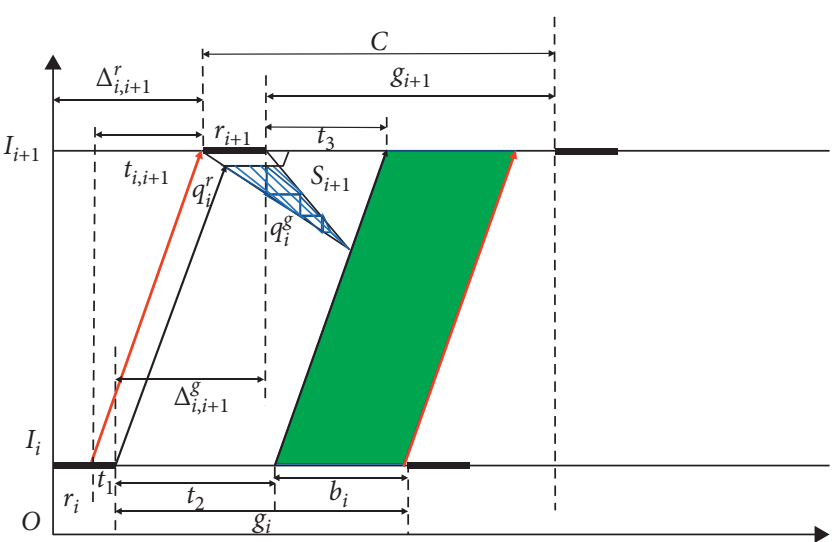

(b)

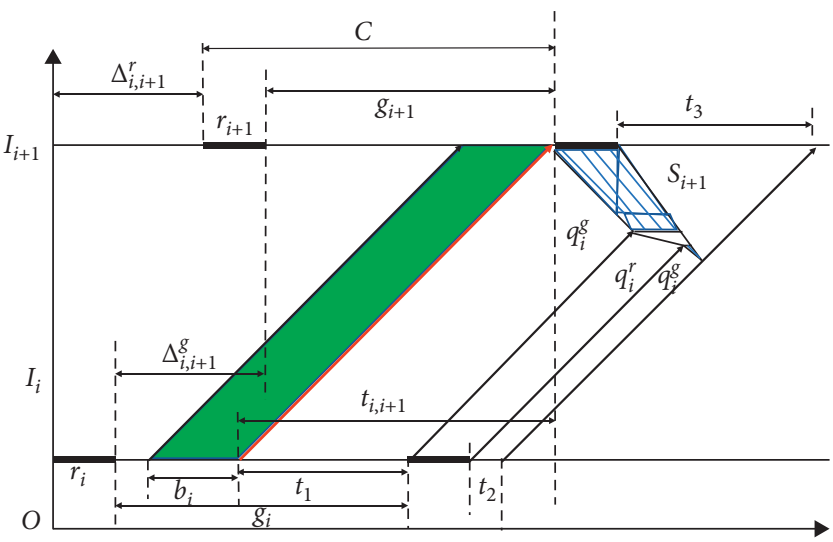

(d)

Figure 2: Four categories of interlaced spatiotemporal subplots.

the $i+1$ th intersection, the front part of them should wait. We denote $t_{1}$ as the max time interval when vehicles leave from $i$ th intersection and wait at the next node. $t_{2}$ denotes the waiting time of the foremost vehicle from the progression band, and $t_{3}$ denotes the congestion evacuation time. $D$ also denotes the traffic delay of vehicles from the progression band, which is represented by the shadow part in this figure. We then derive the following:

$$
\begin{aligned}
& \left\{\begin{array}{l}
t_{1} q_{i}^{r}+t_{2} q_{i}^{g}=t_{3} S_{i+1}, \\
t_{3}+r_{i+1}=t_{1}+t_{2}, \\
t_{1}=r_{i}-\Delta_{i, i+1}^{r}+t_{i, i+1}, \\
b_{i}=g_{i}-t_{2},
\end{array}\right. \\
& b_{i}=g_{i}-\frac{\left(r_{i}-\Delta_{i, i+1}^{r}+t_{i, i+1}\right)\left(q_{i}^{r}-S_{i+1}\right)+r_{i+1} S_{i+1}}{S_{i+1}-q_{i}^{g}}, \\
& D=\frac{t_{2} q_{i}^{g}}{2}\left(\frac{t_{1} q_{i}^{r}}{S_{i+1}}+\Delta_{i, i+1}^{g}-t_{i, i+1}\right) \\
& \quad=\frac{\left(r_{i}-\Delta_{i, i+1}^{r}+t_{i, i+1}\right)\left(q_{i}^{r}-S_{i+1}\right)+r_{i+1} S_{i+1}}{2\left(S_{i+1}-q_{i}^{g}\right)} q_{i}^{g}\left(\frac{t_{1} q_{i}^{r}}{S_{i+1}}+\Delta_{i, i+1}^{r}+r_{i+1}-r_{i}-t_{i, i+1}\right) .
\end{aligned}
$$


Category III $\left(\Delta_{i, i+1}^{r}<t_{i, i+1}<C\right.$ (Figure 1 part (3)) $)$ : in Figure 2(c), at this time, the vehicle which reaches at the start time of the next intersection at the specific speed is at the range of green time at $i$ th intersection, and the tail of the traffic flow in the progression band must be congested, and the front of the vehicle should wait or not wait depending on evacuation rate at $i+1$ th node. At this time, it is divided into two categories. This one discusses that the saturated flow rate is large enough that the congestion has vanished when the head of the progression band passes. We denote $t_{1}$ as the max time interval when vehicles leave from $i$ th intersection and wait at the next node. $t_{2}$ denotes the waiting time of the end vehicle from the progression band, and $t_{3}$ denotes the congestion evacuation time, $t^{\prime}$ denotes threshold time between block and unblock. $D$ also denotes the traffic delay of vehicles from the progression band, which is represented by the shadow part in this figure. We then derive the following:

$$
\begin{aligned}
& \left\{\begin{array}{l}
r_{i} q_{i}^{r}+t_{2} q_{i}^{g}=t^{\prime} S_{i+1}, \\
t_{3} \leq t^{\prime},
\end{array}\right. \\
& \left\{\begin{array}{l}
t_{1} q_{i}^{r}+t_{2} q_{i}^{g}=t_{3} S_{i+1}, \\
t_{3}+r_{i+1}=t_{1}+t_{2}, \\
b_{i}=g_{i}-t_{2}, \\
t_{2}=t_{i, i+1}-\Delta_{i, i+1}^{r}, \\
b_{i}=g_{i}-t_{i, i+1}+\Delta_{i, i+1}^{r}, \\
D=\frac{t_{2} q_{i}^{g} *\left(r_{i+1}+\frac{t_{2} q_{i}^{g}}{S_{i+1}}+\Delta_{i, i+1}^{g}+r_{i}-t_{i, i+1}\right)}{2} \\
=\frac{t_{2} q_{i}^{g}}{2} *\left(2 r_{i+1}+\frac{t_{2} q_{i}^{g}}{S_{i+1}}+\Delta_{i, i+1}^{r}-t_{i, i+1}\right) .
\end{array}\right.
\end{aligned}
$$

In Figure 2(d), when the saturated flow rate is not enough, the head of the progression band from ith intersection should wait. $t_{1}$ denotes the green time interval of the tail of traffic flow at $i$ th intersection, $t_{2}$ denotes the green time interval of the head of traffic flow at $i$ th intersection, $t_{3}$ denotes the congestion evacuation time, and $t^{\prime}$ denotes threshold time between block and unblock.

$$
\begin{aligned}
& \left\{\begin{array}{l}
r_{i} q_{i}^{r}+t_{1} q_{i}^{g}=t^{\prime} S_{i+1}, \\
t_{3}>t^{\prime}
\end{array}\right. \\
& \left\{\begin{array}{l}
t_{1} q_{i}^{g}+r_{i} q_{i}^{r}+t_{2} q_{i}^{g}=t_{3} S_{i+1}, \\
t_{3}=t_{2}+t_{i, i+1}+r_{i}-\Delta_{i, i+1}^{r}-r_{i+1}, \\
t_{1}=t_{i, i+1}-\Delta_{i, i+1}^{r}, \\
b_{i}=g_{i}-t_{1}-t_{2}, \\
b_{i}=g_{i}-t_{i, i+1}+\Delta_{i, i+1}^{r} \\
\quad-\frac{\left(t_{i, i+1}-\Delta_{i, i+1}^{r}\right) q_{i}^{g}+r_{i} q_{i}^{r}-\left(t_{i, i+1}+r_{i}-\Delta_{i, i+1}^{r}-r_{i+1}\right) S_{i+1}}{S_{i+1}-q_{i}^{g}} \\
+\frac{t_{2} q_{i}^{g}}{2}\left(\frac{t_{1} q_{i}^{g}+r_{i} q_{i}^{r}}{S_{i+1}}+\Delta_{i, i+1}^{g}-t_{i, i+1}\right) \\
D= \\
\quad \frac{t_{1} q_{i}^{g}}{2}\left(\frac{t_{1} q_{i}^{g}}{S_{i+1}}+2 r_{i+1}+\Delta_{i, i+1}^{r}-t_{i, i+1}\right)
\end{array}\right.
\end{aligned}
$$

In Figure 3, when $\Delta_{i, i+1}^{r}<r_{i}, \Delta_{i, i+1}^{g}<g_{i}$, according to the position of the last intersection corresponding to the start time of the red light at the next intersection, it is divided into three parts of four categories. The slope of red lines in Figure 4 is the basis of classification.

Category I $\left(t_{i, i+1}<\Delta_{i, i+1}^{r}\right.$ (Figure 3 part (1))): in Figure 4(a), this situation is the same as category II in the interlaced one (Figure 2(b)). So the notions are omitted, and equation (3) should be used.

Category II $\left(\Delta_{i, i+1}^{r}<t_{i, i+1}<\Delta_{i, i+1}^{r}+g_{i}\right.$ (Figure 3 part (2))): in Figures $4(\mathrm{~b})$ and $4(\mathrm{c})$, this situation is the same as category III in the interlaced one (Figures 2(c) and $2(\mathrm{~d}))$, So the notions are omitted, and equation (4) and (5) should be used.

Category III $\left(\Delta_{i, i+1}^{r}+g_{i}<t_{i, i+1}<C\right.$ (Figure 3 part (3))): in Figure 4(d), under the specific speed, the vehicles from the $i$ th intersection of the progression band must wait at the next intersection, so it can generate green wave, which means that this situation is eliminated.

In Figure 5, when $\Delta_{i, i+1}^{r}>r_{i}, \Delta_{i, i+1}^{g}>g_{i}$, according to the vehicle's position at the last node which reaches at the start 


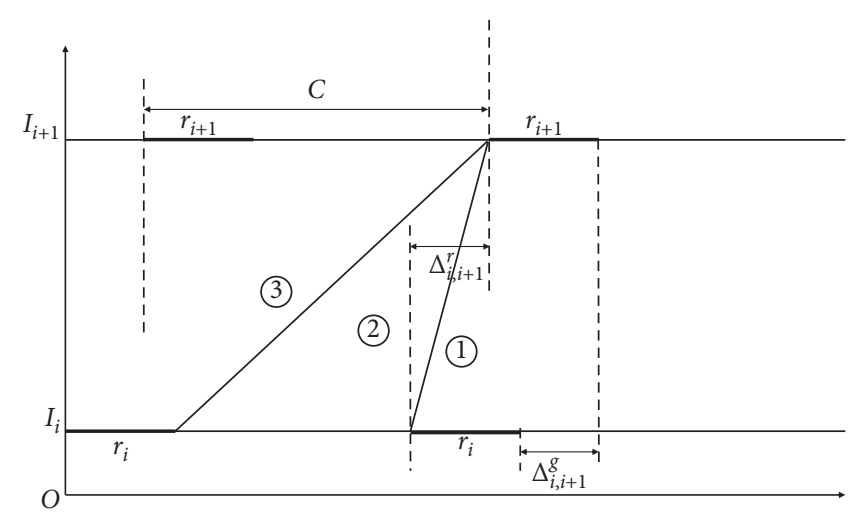

Figure 3: Classification of forward overlapping spatiotemporal figures according to offset.

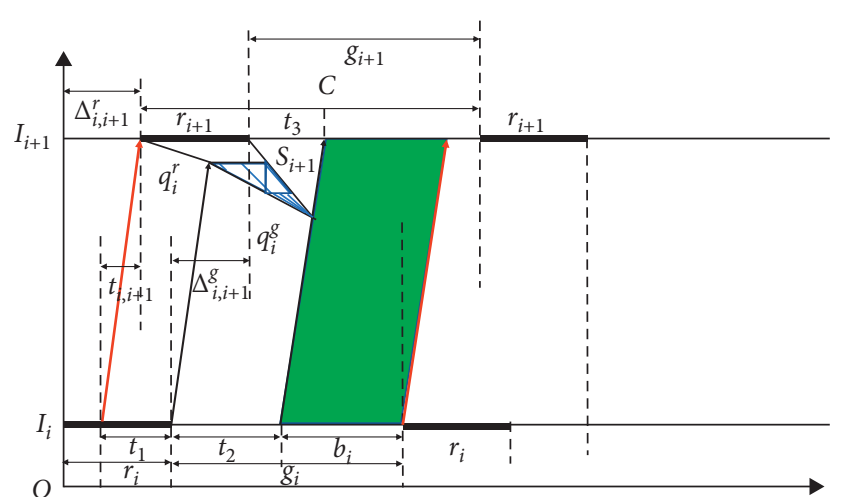

(a)

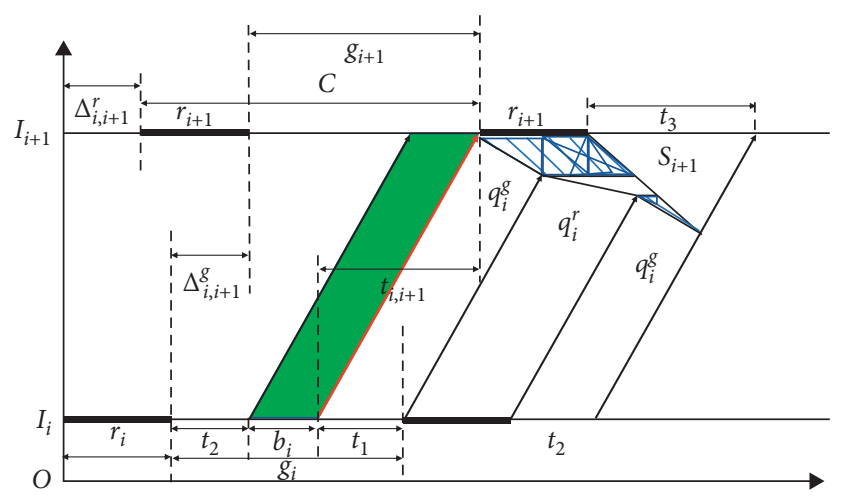

(c)

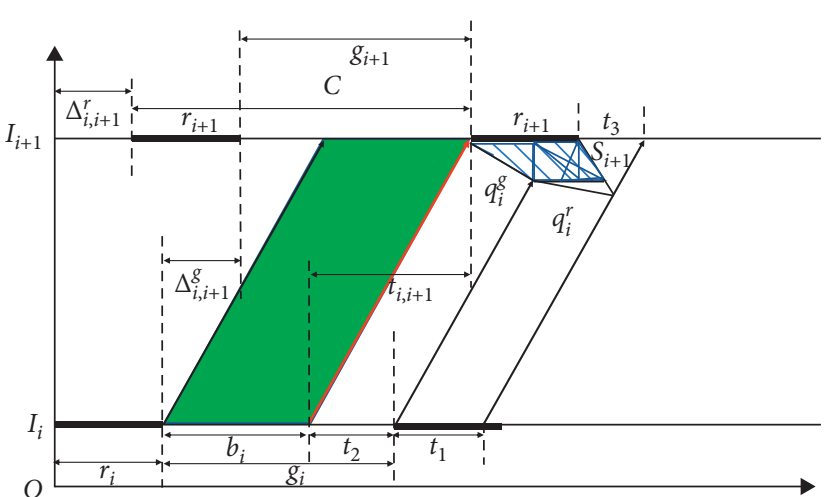

(b)

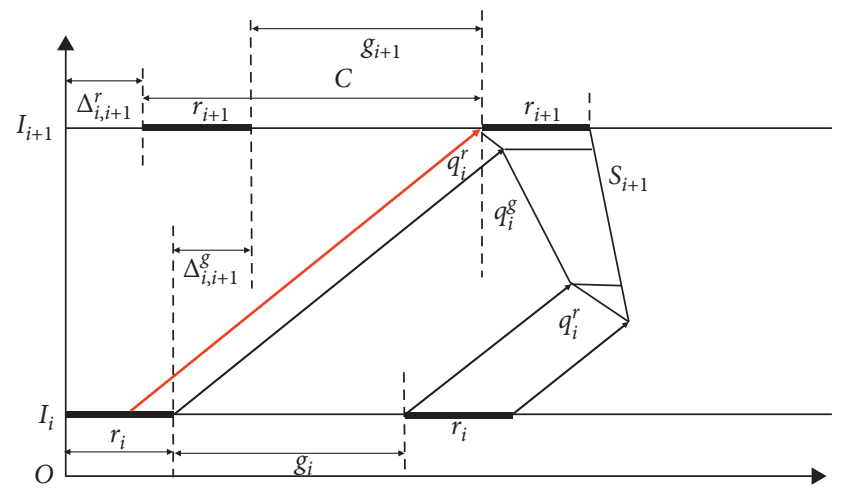

(d)

FIGURE 4: Four categories of forward overlapping spatiotemporal subplots.

time of red at the next intersection, it is divided into three parts of five categories. The slope of red lines in Figure 6 is the basis of classification.

Category I $\left(t_{i, i+1}<\Delta_{i, i+1}^{r}-r_{i}\right.$ (Figure 5 part (1))): in Figure 6(a), at this time, the vehicle which reaches at the start time of the next intersection at the specific speed is at the range of green time at $i$ th intersection, and the tail of the traffic flow in the progression band must be congested, and the front of the vehicle should or should not wait depending on evacuation rate at $i+1$ th node.
At this time, it is divided into two categories. This one discusses that the saturated flow rate is large enough that the congestion has vanished when the head of the progression band passes. We denote $t_{1}$ as the max time interval when vehicles leave from $i$ th intersection and wait at the next node. $t_{2}$ denotes the waiting time of the end vehicle from progression band, $t_{3}$ denotes the congestion evacuation time, and $t^{\prime}$ denotes threshold time between block and unblock. $D$ also denotes the traffic delay of vehicles from the progression band, 


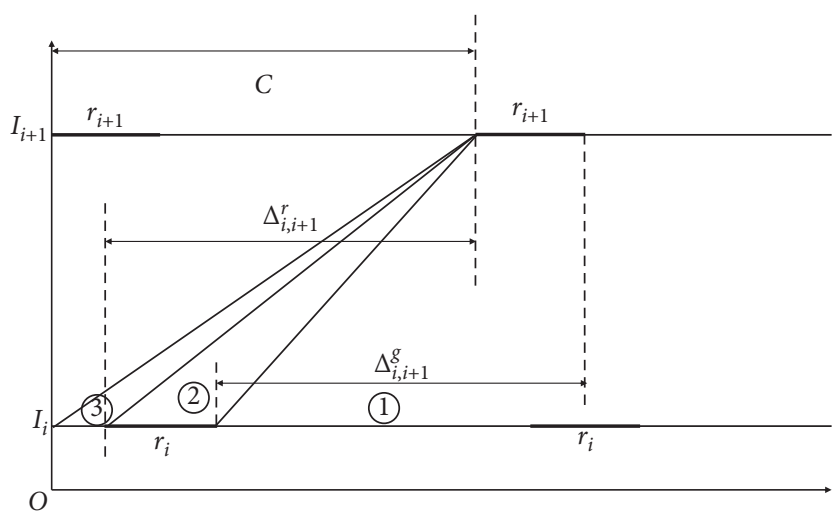

Figure 5: Classification of backward overlapping spatiotemporal figures according to offset.

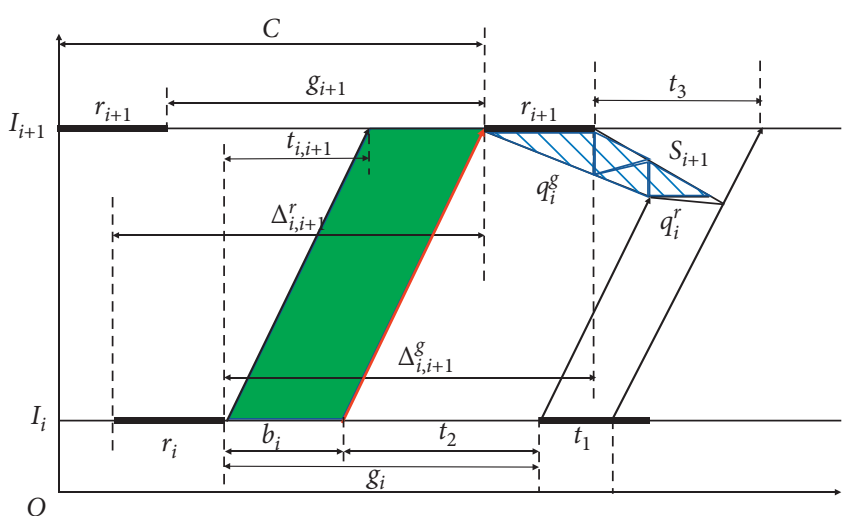

(a)

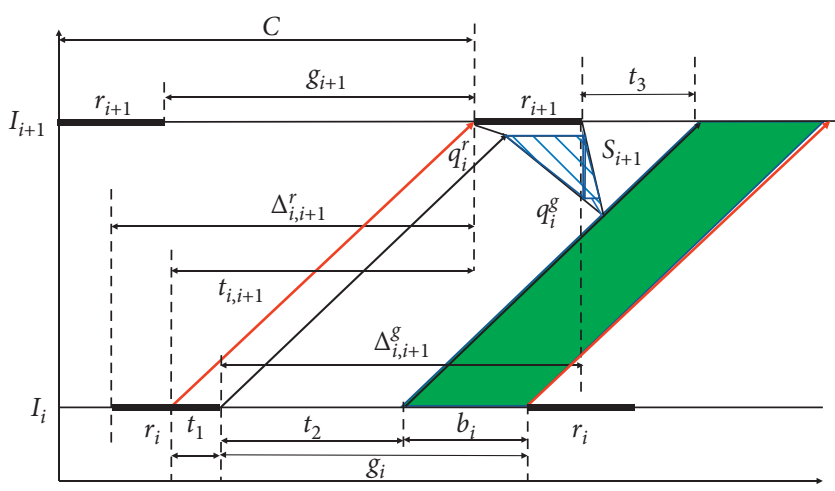

(c)

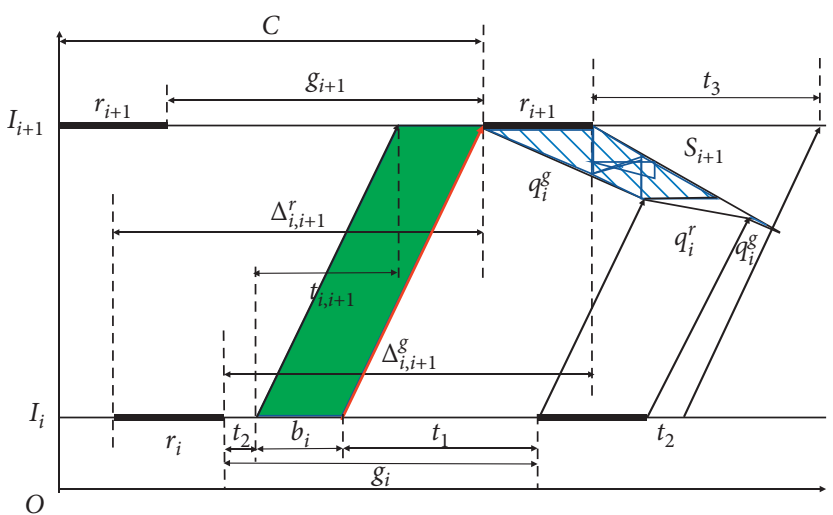

(b)

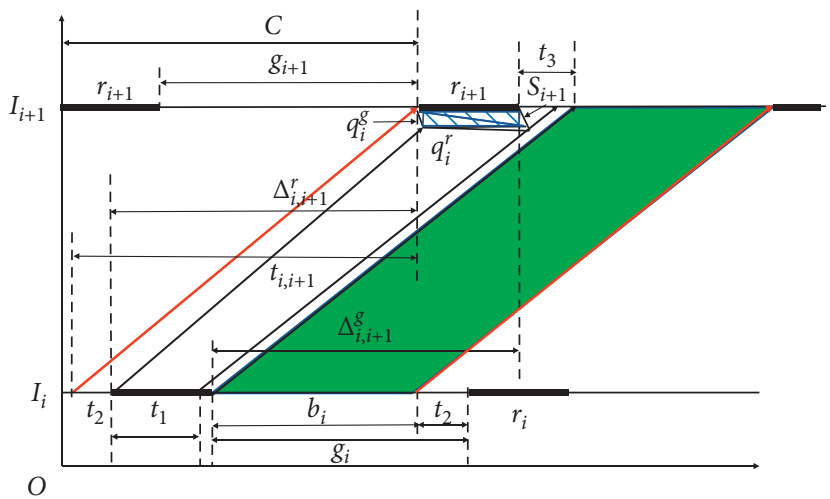

(d)

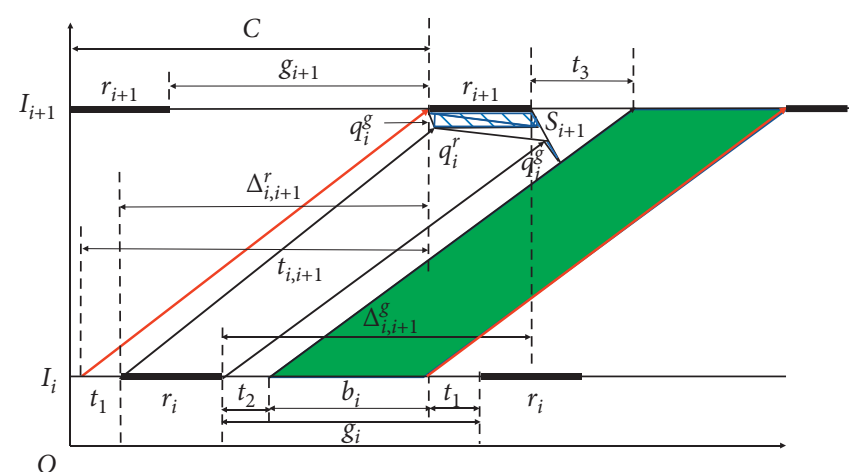

(e)

FIGURE 6: Five categories of backward overlapping spatiotemporal subplots. 
which is represented by the shadow part in this figure. We then derive the following:

$$
\begin{aligned}
& \left\{\begin{array}{l}
r_{i} q_{i}^{r}+t_{2} q_{i}^{g}=t / S_{i+1}, \\
t_{3} \leq t \prime
\end{array}\right. \\
& \left\{\begin{array}{l}
t_{1} q_{i}^{r}+t_{2} q_{i}^{g}=t_{3} S_{i+1}, \\
t_{3}+r_{i+1}=t_{1}+t_{2}, \\
b_{i}=g_{i}-t_{2}, \\
t_{2}=t_{i, i+1}-\Delta_{i, i+1}^{r}+C,
\end{array}\right. \\
& b_{i}=g_{i}-t_{i, i+1}+\Delta_{i, i+1}^{r}-C, \\
& D=\frac{t_{2} q_{i}^{g}}{2} *\left(r_{i+1}+\frac{t_{2} q_{i}^{g}}{S_{i+1}}+\Delta_{i, i+1}^{g}-g_{i}-t_{i, i+1}\right) \\
& =\frac{t_{2} q_{i}^{g}}{2} *\left(2 r_{i+1}+\frac{t_{2} q_{i}^{g}}{S_{i+1}}+\Delta_{i, i+1}^{r}-C-t_{i, i+1}\right) .
\end{aligned}
$$

In Figure 6(b), when the saturated flow rate is not enough, the head of the progression band from ith intersection should wait. $t_{1}$ denotes the green time interval of the tail of traffic flow at ith intersection, $t_{2}$ denotes the green time interval of the head of traffic flow at $i$ th intersection, $t_{3}$ denotes the congestion evacuation time, and $t^{\prime}$ denotes threshold time between block and unblock.

$$
\begin{aligned}
& \left\{\begin{array}{l}
r_{i} q_{i}^{r}+t_{1} q_{i}^{g}=t / S_{i+1} \\
t_{3}>t \prime
\end{array}\right. \\
& \left\{\begin{array}{l}
t_{1} q_{i}^{g}+r_{i} q_{i}^{r}+t_{2} q_{i}^{g}=t_{3} S_{i+1}, \\
t_{3}=t_{2}+t_{i, i+1}+r_{i}-\Delta_{i, i+1}^{r}-r_{i+1}+C, \\
t_{1}=t_{i, i+1}-\Delta_{i, i+1}^{r}+C, \\
b_{i}=g_{i}-t_{1}-t_{2},
\end{array}\right. \\
& b_{i}=g_{i}-t_{i, i+1}-C+\Delta_{i, i+1}^{r} \\
& -\frac{\left(t_{i, i+1}+C-\Delta_{i, i+1}^{r}\right) q_{i}^{g}+r_{i} q_{i}^{r}-\left(t_{i, i+1}+r_{i}+C-\Delta_{i, i+1}^{r}-r_{i+1}\right) S_{i+1}}{S_{i+1}-q_{i}^{g}}, \\
& D=\frac{t_{1} q_{i}^{g}}{2}\left(\frac{t_{1} q_{i}^{g}}{S_{i+1}}+2 r_{i+1}+\Delta_{i, i+1}^{r}-C-t_{i, i+1}\right) \\
& +\frac{t_{2} q_{i}^{g}}{2}\left(\frac{t_{1} q_{i}^{g}+r_{i} q_{i}^{r}}{S_{i+1}}-C+\Delta_{i, i+1}^{g}-t_{i, i+1}\right) .
\end{aligned}
$$

Category II $\left(\Delta_{i, i+1}^{r}-r_{i}<t_{i, i+1}<\Delta_{i, i+1}^{r}\right.$ (Figure 5 part (2))): in Figure 6(c), this situation is the same as category II in the interlaced one (Figure 2(b)). So the notions are omitted, and equation (3) should be used.

Category III $\left(\Delta_{i, i+1}^{r}<t_{i, i+1}<C\right.$ (Figure 5 part (3)) : in Figures $6(\mathrm{~d})$ and $6(\mathrm{e})$, this situation is the same as the category III in the interlaced one (Figures 2(c) and $2(d)$ ), So the notions are omitted, and equations (4) and (5) should be used.

Finally, we have proposed progression bandwidth models considering different offset, green time, and average travel time, which is summarized in Table 2.

Above all, we get the progression bandwidth and motor vehicle delay of adjacent intersections in each case, and finally, there will be multiple progression bandwidths on different road sections. At present, the optimization methods for coordinated control of traffic signals are the maximum progression bandwidth method and the minimum delay method.

The maximum progression bandwidth method is to ensure that the vehicle (vehicle flow) can occupy the longest period of one cycle through the intersection in a continuous manner. Since the cycle length of each coordinated intersection is assumed to be the same, the progression band is satisfied here. Of course, this will ensure that the most traffic flow can participate in coordinated control. For the case where the length of the progression band is different in different sections, the minimum value is taken as the inspection index. The minimum delay method is to minimize the sum of the delays of the progression motor vehicle at the intersection (the delay of the nongreen wave vehicle is not considered here), which is another reasonable solution in theory. Delays are not only related to coordinated control but also related to vehicle emissions, parking, fuel consumption, and so forth, thus affecting the corresponding driving costs.

In addition, in the equations of the aforementioned models, some models' bandwidths are negatively correlated with delays, and some models satisfy the quadratic function of the two variables, which is relatively complicated. When the progression bandwidth is the largest, the motor vehicle delay is not necessarily the smallest; otherwise, when the motor vehicle delay is the smallest, the progression bandwidth is not necessarily the largest. In addition to the pedestrian delays to be studied after this article, there are at least three evaluation indicators, as for how to assign weights, how to give comprehensive evaluation indicators reasonably, and then give them in detail in Section 4.

\section{Model of Pedestrian Delay}

Since this study not only considers the coordinated control of traffic signals, but also considers a large number of pedestrian flows, it is necessary to consider not only the coordination and optimization of the progression bands at each intersection, but also the minimization of pedestrian crossing delays. In fact, signal control interacts with pedestrians crossing the street. Pedestrians will affect the pedestrian clearing time at the intersection, thus affecting the green time of the opposite vehicle. Too many vehicles will 
TABLE 2: Summary of progression bandwidth models.

\begin{tabular}{llccc}
\hline & Conditions & Analysis position (ith node) & Congestion situation & Equation \\
\hline$\Delta_{i, i+1}^{g}<g_{i}$ & $t_{i, i+1}<\Delta_{i, i+1}^{r}-r_{i}$ & Mid & $(2)$ \\
$\Delta_{i, i+1}^{r}>r_{i}$ & $\Delta_{i, i+1}^{r}-i<t_{i, i+1}<\Delta_{i, i+1}^{r}$ & Green-green & Head & $(3)$ \\
& $\Delta_{i, i+1}^{r}<t_{i, i+1}<C$ & Red-green & Tail & $(4)$ \\
\hline & $t_{i, i+1}<\Delta_{i, i+1}^{r}<\Delta_{i, i+1}^{r}+g_{i}$ & Green-red-green & Head and tail & $(5)$ \\
$\Delta_{i, i+1}^{g}<g_{i}$ & $\Delta_{i, i+1}^{r}<t_{i, i+1}^{r}<t_{i, i}^{r}$ & Head & $(3)$ \\
$\Delta_{i, i+1}^{r}>r_{i}$ & $\Delta_{i, i+1}^{r}+g_{i}<t_{i, i+1}<C$ & Red-green & Tail & $(4)$ \\
& & Reen-gred-green & Head and tail & $(5)$ \\
\hline & $t_{i, i+1}^{r}<\Delta_{i, i+1}^{r}-r_{i}$ & All flow & Tail & $(6)$ \\
$\Delta_{i, i+1}^{g}<g_{i}$ & $\Delta_{i, i+1}^{r}-r_{i}<t_{i, i+1}<\Delta_{i, i+1}^{r}$ & Green-red-green & Head and tail & $(7)$ \\
$\Delta_{i, i+1}^{r}>r_{i}$ & $\Delta_{i, i+1}^{r}<t_{i, i+1}<C$ & Red-green & Head & $(3)$ \\
& & Green-red-green & Tail & $(4)$ \\
& & & Head and tail & $(5)$ \\
\hline
\end{tabular}

aggravate their conflict with pedestrians, resulting in increasing delays. In addition, there have been many studies on the delays of pedestrians at the intersection of signal timing, so we should consider the degree of adaptation of different models in different specific situations.

The specific process of this chapter is as follows: firstly, we introduce several pedestrian delay models, analyze their advantages and disadvantages, applicable conditions, and then use Vissim microsimulation to obtain three cases of motor vehicles through cluster analysis, simulating pedestrian flow from less to more. Then the simulation results are compared with the calculation results, and the errors are calculated to determine the adaptability of each model under different flow rates, which lays a theoretical foundation for the actual analysis of pedestrian flow in the following section.

\subsection{Different Models of Pedestrian Delay}

\subsubsection{HCM2000}

$$
d=\frac{(C-G)^{2}}{2 C} .
$$

where $C$ denotes cycle length of intersection(s), $G$ denotes green time of a cycle(s). The model is based on the equal pedestrian arrival rate, the same signal period, and there is no conflict between pedestrians and motor vehicles. It is an ideal model which is applicable to intersections where motor vehicles and pedestrian traffic are small, which is not suitable for the background of this project. Nevertheless, a series of improved models based on this model have been promoted even more.

\subsubsection{BR Model [33]}

$$
d=F \frac{\left(C-G^{2}\right)}{2 C},
$$

where $F$ denotes the proportion of pedestrians obeying traffic rules. This model takes into account pedestrians who do not follow traffic rules and believes they will not be delayed. Especially suitable for the situation in developing areas, people generally have a weak sense of traffic. However, the model does not consider the conflict between pedestrians and motor vehicles, and there is no clear behavioral assumption. The applicable conditions are subject to great constraints.

\subsubsection{Model [34].}

$$
\begin{aligned}
& D=d_{G}+\frac{k_{\mathrm{NU}} R_{E}^{2} k}{2 C}, \\
& k=1-[1-(-0.08+0.90 q)] P_{w} .
\end{aligned}
$$

where $d_{G}$ denotes average delays of pedestrians(s), $k_{\mathrm{NU}}$ denotes adjustment factor of uneven arrival rate, $R_{E}$ denotes effective red time(s), $P_{w}$ denotes the proportion of violations, and $q$ denotes average arrival rate of vehicles $(\mathrm{pcu} / \mathrm{s})$. This model assumes that the pedestrian arrival rate is not fixed throughout a cycle and that pedestrians arriving in the green time and pedestrians who do not obey the traffic rules will still have delays. Furthermore, the average delay of pedestrians varies with the entire cycle, and these changes are certain. At the green time, the average delay of pedestrians is fixed. At the beginning of the red time, the value is the largest, then linearly decreases, and periodically changes. This model is not suitable for areas where pedestrian flow is high, and the calculated value will be small.

\subsubsection{Z Model [35].}

$$
\begin{aligned}
d & =d_{j}+d_{c} \\
& =\frac{G^{2} s}{2 C(s-q)}+\frac{\left(1 / \lambda_{1}\right)-\left(\tau+\left(1 / \lambda_{1}\right)\right) e^{-\tau\left(\lambda_{1}+\lambda_{2}\right)}}{e^{-\tau\left(\lambda_{1}+\lambda_{2}\right)}}, \\
\tau & =\frac{W}{V_{p}}+R+l .
\end{aligned}
$$

where $s$ denotes saturated pedestrian flow (ped/h), $q$ denotes average arrival rate of pedestrians (ped/h), $\lambda_{1}$ denotes arrival 
rate of left-turn vehicles ( $\mathrm{pcu} / \mathrm{s}), \lambda_{2}$ denotes arrival rate of right-turn vehicles (pcu/s), $\tau$ denotes safety gap(s), $W$ denotes width of motorway $(\mathrm{m}), V_{p}$ denotes average speed of pedestrians $(\mathrm{m} / \mathrm{s}), R$ denotes reaction time $(\mathrm{s})$, and $l$ denotes time through vehicle(s). This model considers the delays of pedestrians waiting at the red light and also takes the delays caused by left- or right-turning vehicles as they cross the crosswalk into account.

\subsubsection{Model [36].}

$$
\begin{aligned}
D_{\mathrm{Avg}} & =D_{\mathrm{WT}}+D_{\mathrm{CT}}+D_{\mathrm{VIT}} \\
& =\frac{\alpha_{1}\left(C-\left[G+\alpha_{2} R\right]\right)^{2}}{2 C}+\Delta_{t}(\gamma-1)+D_{\mathrm{VIT}} .
\end{aligned}
$$

where $\alpha_{1}$ denotes adjustment factor of uneven arrival rate, $\alpha_{2}$ denotes pedestrian ratio of noncompliance with traffic regulations, $\Delta_{t}$ denotes ideal time of $\operatorname{crossing}(\mathrm{s}), \gamma$ denotes adjustment factor of pedestrian crossing, $D_{\mathrm{VIT}}$ denotes conflict delay of pedestrian and vehicle(s). The model actually has three components, including pedestrian waiting time delay, transit time delay, and conflict delay with the motor vehicle. The pedestrian waiting time delay is obtained by correcting HCM delay formula. The transit time delay is obtained by the speed expansion theory. The third part is obtained through a binary logit model, which was obtained by directly looking up the table during the project. The model is suitable for complex intersections and assumes that the pedestrian arrival rate is uneven; pedestrians will violate traffic rules, pedestrian speed changes, and pedestrian-vehicle conflicts.

3.2. Adaptive Analysis. We have obtained five different models from the literature review, and we have two variables: vehicles flow and pedestrian flow. To make it simple, we discrete them, that is, take a value every 50 and 100, respectively, in Vissim simulation. However, we also need a dimensionality reduction. In this study, we use cluster analysis. For more information, please see the appendix. Here we directly show the results. The relationship between pedestrian delay and motor vehicle flow is not significant, while significant in pedestrian flow. According to the flow rate of the motor vehicle, it is divided into three categories, that is, $q=600 \mathrm{pcu} / \mathrm{h}$ when the motor flow is small; $q=1500 \mathrm{pcu} / \mathrm{h}$ when the motor flow is moderate; $q=2400 \mathrm{pcu} / \mathrm{h}$ when the motor flow is large.

Here are the details in Figure 7. There are six subplots, in the left column are the pedestrian delay(s) as the function of pedestrian flow (ped/h), in the right column are the relative error as the function of pedestrian flow (ped/h). In the first row, the two figures depict the situation under small vehicle flow $(q=600 \mathrm{pcu} / \mathrm{h})$. In the second row, the two figures depict the situation under moderate vehicle flow ( $q=1500 \mathrm{pcu} / \mathrm{h})$. In the third row, the two figures depict the situation under large vehicle flow $(q=2400 \mathrm{pcu} / \mathrm{h})$. The gray (only in the left column) lines denote delay in the simulation, the yellow lines denote delay and relative error of HCM model, the red lines denote delay and relative error of BR model, the green lines denote delay and relative error of LI model, the blue lines denote delay and relative error of $\mathrm{Z}$ model, and the purple lines denote delay and relative error of MV model.

In Figures 7(a) and 7(b), we find that when the arrival rate of pedestrians is less than $600 \mathrm{ped} / \mathrm{h}$; HCM model is more in line with this situation. When the arrival rate of pedestrians is between $600 \mathrm{ped} / \mathrm{h}$ and $1200 \mathrm{ped} / \mathrm{h}$, LI model is more accurate because as the pedestrian flow increases, there are more people who do not obey the rules, and these people can also generate delays. When the flow rate exceeds $1200 \mathrm{ped} / \mathrm{h}$, the MV model fits well, because when pedestrians increase, the conflict with the motor vehicle increases gradually (although the motor vehicle flow is small). At this time, both the MV model and the $\mathrm{Z}$ model consider the conflict between pedestrians and motor vehicles. However, since the $\mathrm{Z}$ model is limited by the maximum flow of pedestrians, the error near the maximum flow is significantly large, and the conflict between pedestrians and motor vehicles in the MV model should be obtained from many curves of surveys, so the fault tolerance rate is higher.

In Figures 7(c) and 7(d), when the pedestrian flow is less than $1200 \mathrm{ped} / \mathrm{h}$, the LI model has a good fitting degree. When the pedestrian flow is greater than $1200 \mathrm{ped} / \mathrm{h}$, the MV model has a high degree of fitting. While other models are not suitable under these conditions, especially unlike before, the HCM model has a low fit at low flow rates. This is because the delay model just only considers cycle length and green time as the vehicle flow increases. There are also a number of correction factors to consider, including pedestrian behavioral factors, delays caused by pedestrians and motor vehicle conflicts. These two models take the above effects into account.

In Figures 7(e) and 7(f), when the pedestrian flow is less than $900 \mathrm{ped} / \mathrm{h}$, the $\mathrm{Z}$ model has a higher degree of fitting. When the pedestrian flow is between 900-1600 ped/h, the LI model fits to a higher degree. When the pedestrian flow is greater than $1600 \mathrm{ped} / \mathrm{h}$, only the MV model can be used. This is because when the pedestrian flow is small, the MV model overconsiders the delay caused by the conflict between the motor vehicle and the pedestrians, so the error is relatively large; on the contrary, when the pedestrian flow is large, the collision between the pedestrian and the car is gradually intensified. This should be taken into consideration, and the $\mathrm{Z}$ model is nonlinearly increasing due to the limitation of the pedestrian flow, and the growth rate is gradually increasing. Therefore, the $\mathrm{Z}$ model is no longer applicable when approaching the upper limit of pedestrian flow. At the same time, the LI model takes care of both. 


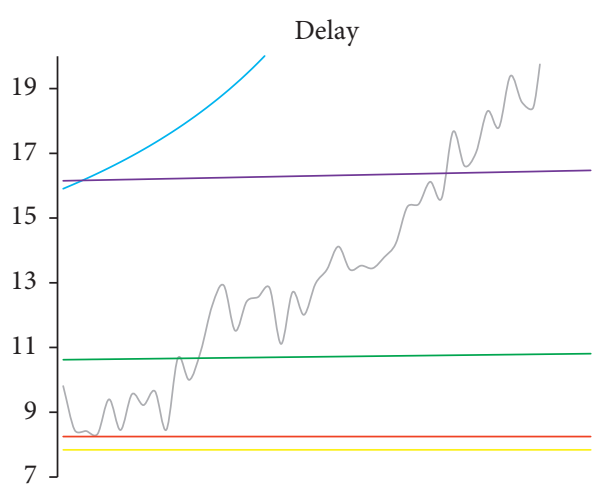

(a)

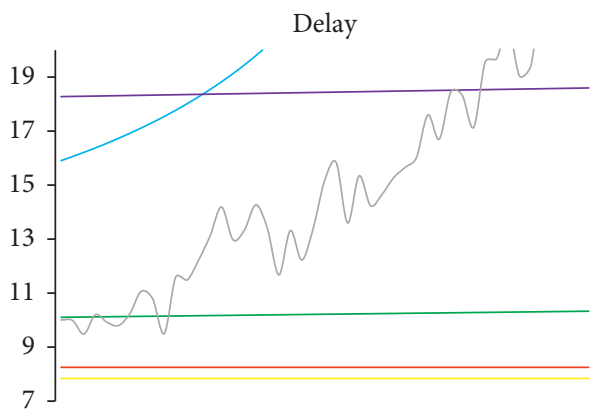

(c)

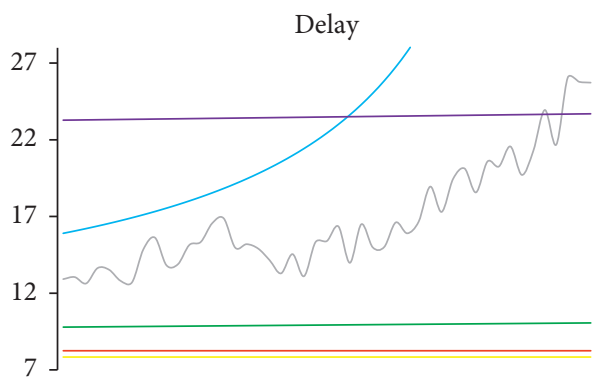

(e)

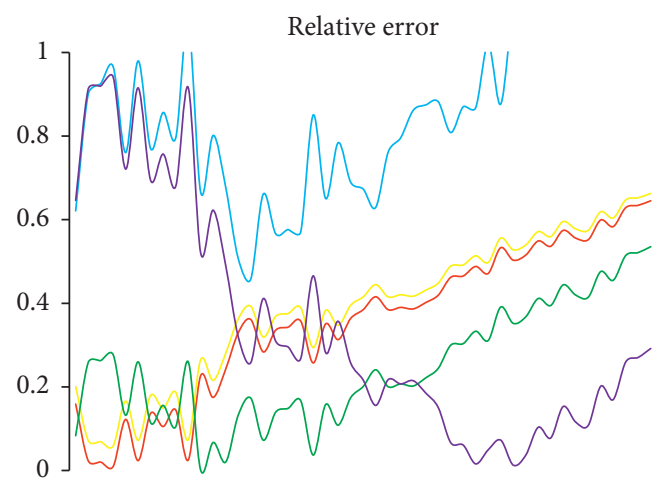

(b)

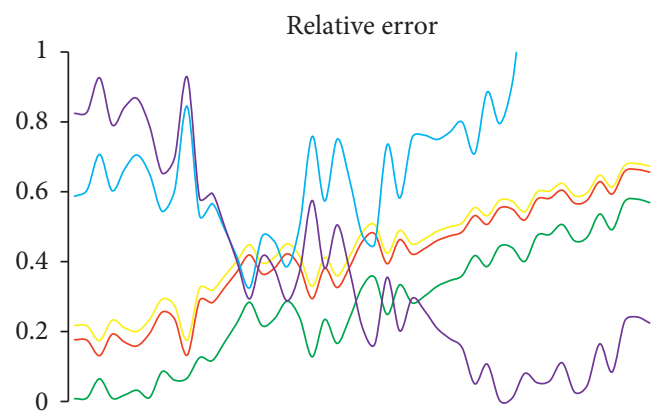

(d)

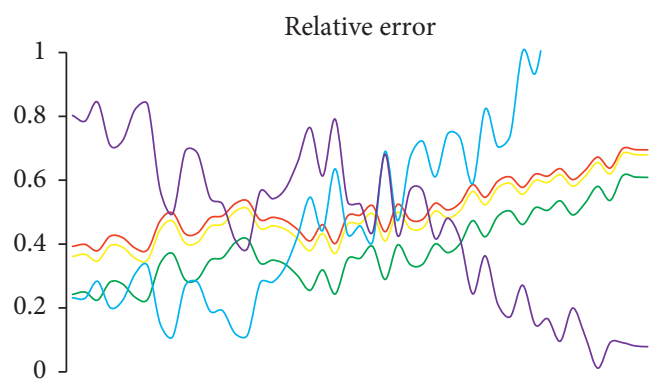

(f)

FIGURE 7: Delay and relative error of different models compared with simulation results.

Finally, we summarize the equations of calculating pedestrian delay as follows:

$$
\left\{\begin{array}{l}
q_{v}<900 \mathrm{pcu} / \mathrm{h} \begin{cases}\mathrm{HCM}, & q_{p}<600 \mathrm{peh} / \mathrm{h}, \\
\mathrm{LI}, & 600 \mathrm{peh} / \mathrm{h} \leq q_{p}<1200 \mathrm{ped} / \mathrm{h}, \\
\mathrm{MV}, & 1200 \mathrm{ped} / \mathrm{h} \leq q_{p},\end{cases} \\
900 \mathrm{pcu} / \mathrm{h} \leq q_{v}<2000 \mathrm{pcu} / \mathrm{h} \begin{cases}\mathrm{LI}, & q_{p}<1200 \mathrm{ped} / \mathrm{h}, \\
\mathrm{MV}, \quad 1200 \mathrm{ped} / \mathrm{h} \leq q_{p},\end{cases} \\
2000 \mathrm{pcu} / \mathrm{h} \leq q_{v} \begin{cases}\mathrm{Z}, & q_{p}<900 \mathrm{ped} / \mathrm{h}, \\
\mathrm{LI}, & 900 \mathrm{ped} / \mathrm{h} \leq q_{p}<1600 \mathrm{ped} / \mathrm{h}, \\
\mathrm{MV}, & 1600 \mathrm{ped} / \mathrm{h} \leq q_{p} .\end{cases}
\end{array}\right.
$$

\section{Algorithm and Case Study}

From Sections 2 and 3, we have established coordinated control progression band models and pedestrian delay models according to different actual situations. The most crucial point of this project is to combine the two models. For example, in the vicinity of the subway station, it is necessary to consider not only the optimization of coordinated control, but also the large number of pedestrian flows periodically entering and leaving the subway station. Firstly, we propose an algorithm to deal with this bilevel problem and then use a case to test the rationality of models and this algorithm.

4.1. Bilevel Programming and Algorithm. From the above analysis, the problem can be transformed into the following: 


$$
\begin{array}{ll}
\min & f\left(C, g_{1}, g_{2}, \ldots, g_{n}, q\right) \\
\text { s.t. } & G_{1}\left(C, g_{1}, g_{2}, \ldots, g_{n}, q\right) \subset U, \\
\max & f_{1}\left(C, g_{1}, g_{2}, \ldots, g_{n}, p\right) \\
\min & f_{2}\left(C, g_{1}, g_{2}, \ldots, g_{n}, p\right) \\
\text { s.t. } & G_{2}\left(C, g_{1}, g_{2}, \ldots, g_{n}, p\right) \subset U_{2} .
\end{array}
$$

Since the study focuses on the delay of pedestrian crossing, the minimization of pedestrian delay is defined as the objective function of the upper planning, where $q$ is the other variable related to pedestrian delay except for the cycle length and the green time, which is represented by a letter and it represents a difference from the lower level planning decision variables; the constraints can be expressed as a general formula, specifically the constraints in the actual problem, including the green light time being less than the signal cycle length and within a certain range such as $(C / 4,3 C / 4)$. The lower level planning is a multiobjective nonlinear programming. One is that the progression bandwidth is the largest, and the other is that the total delay of the vehicle at the intersection is the smallest. As with the upper plan, a parameter $p$ is used here to represent the decision variables specific to the underlying plan. The constraints of the lower-level planning are more complicated, including not only the limitation of the green time, but also the constraints of various situations in the model.

It is apparent from the equation that correlating the models of the upper and lower layers by the green time and cycle length has several advantages in the analysis of the problem. There are conflicts and dependencies between the upper and lower layers. Since the road right in the road is certain, the more travel time is given to the pedestrian, the corresponding motor vehicle delay will increase, and the green wave will be difficult to form. Conversely, the smoother the vehicle travels, the less time it takes for pedestrians to cross the street. However, the decision variables in their objective function are repeated and therefore dependent on each other. The decision of the lower layer not only affects the value of its own objective function, but also affects the decision of the upper layer. However, it does not completely unconditionally conform to the upper level plan, and it retains certain autonomy.

There are many bilevel programming solutions, including fuzzy algorithm, branch and bound method, KT method, and pole algorithm. However, the settings are problem-specific under this scenario, like the cycle length is mainly between 60 and 180's as an integer and the green time is positive.If the range of the specific optimal value is not known in advance, it cannot be obtained by branch and bound. And the n-dimensional model can be established only counting the green time at $n$ intersections, and then the offset of adjacent intersections is calculated. The final model must exceed n-dimensional, so the general gradient algorithm and iterative step size algorithm are very difficult to execute. Considering the particularity of the problem, this study turns the double goal into a single objective function as follows:

$$
\begin{aligned}
& Q=\max \frac{\sum_{i} \sum_{j}\left(b_{i j} / d_{i j}^{v}\right)\left(C_{i j}, \Delta_{i j}, g_{i j}\right)}{\sum_{i} \sum_{j} d_{i j}^{p}\left(C_{i j}, g_{i j}, q_{i j}\right)}, \\
& m<C_{i j}<n, \\
& 0 \leq \Delta_{i j}<C_{i j}, \\
& 0 \leq g_{i j}<C .
\end{aligned}
$$

Comparing the five objective functions [37] of traffic signals and cost-effect function proposed by Dr. PeñabaenaNibbles [38], we put forward this final objective function, where $i$ denotes $i$ th intersection, $j$ denotes direction of entrance of intersection, $b_{i j}$ denotes width of progression band, $d_{i j}^{v}$ denotes delays of vehicles, $d_{i j}^{p}$ denotes delays of pedestrians, and $q_{i j}$ denotes flow of vehicles.

The method needs to list the combination of different green times in the same cycle, calculating the corresponding delay and progression bandwidth and the specific computational structure of each layer is shown in Figure 8. Progression bandwidth and motor vehicle delays require three columns as subsystems for the green time and offset between the two intersections and each intersection requires only green time (and other factors which do not change with cycle length) when calculating pedestrian delays. So, each green light column is listed as a subsystem. We then think of it as solving the shortest path problem in the calculation, using the Dijkstra algorithm here. Each subsystem is regarded as the path in the shortest path problem, and the calculated value of the subsystem is the weight. Therefore, the two layers of the problem can be regarded as solving the shortest path problem with $n$ nodes. The pseudocode of the algorithm is in Algorithm 1.

In Algorithm 1, $s, i, j$ denotes node (intersection) and $d$ denotes the total distance of each node. We then suppose an origin and a destination, finding the shortest path between these two nodes. And the corresponding cycle length and offset between the adjacent intersection can be calculated, which means the optimization scheme will be obtained.

4.2. Case Study. This study analyzes the road between Jiming Temple, Fuqiao, and Daxinggong Station of Nanjing Metro Line 3, as shown in Figure 9, namely, Taiping North Road (North-South), Beijing East Road (East-West) from the north and Zhongshan East Road to the south. (Eastwest), with a total length of approximately $1.9 \mathrm{~km}$, with several intersections, including eight signal-controlled intersections. The section is located in the Xuanwu Lake District of Nanjing and is one of the north-south traffic skeleton roads. Judging from the spatial structure of the whole city, Taiping North Road (Beijing East RoadZhongshan East Road) is an important channel for communication between the north and the south of Xuanwu District, which mitigates the main flow of three subway stations to various functional areas; from the surrounding areas in terms of function, the area of Taiping North Road is an important urban block integrating office, residence, study, 


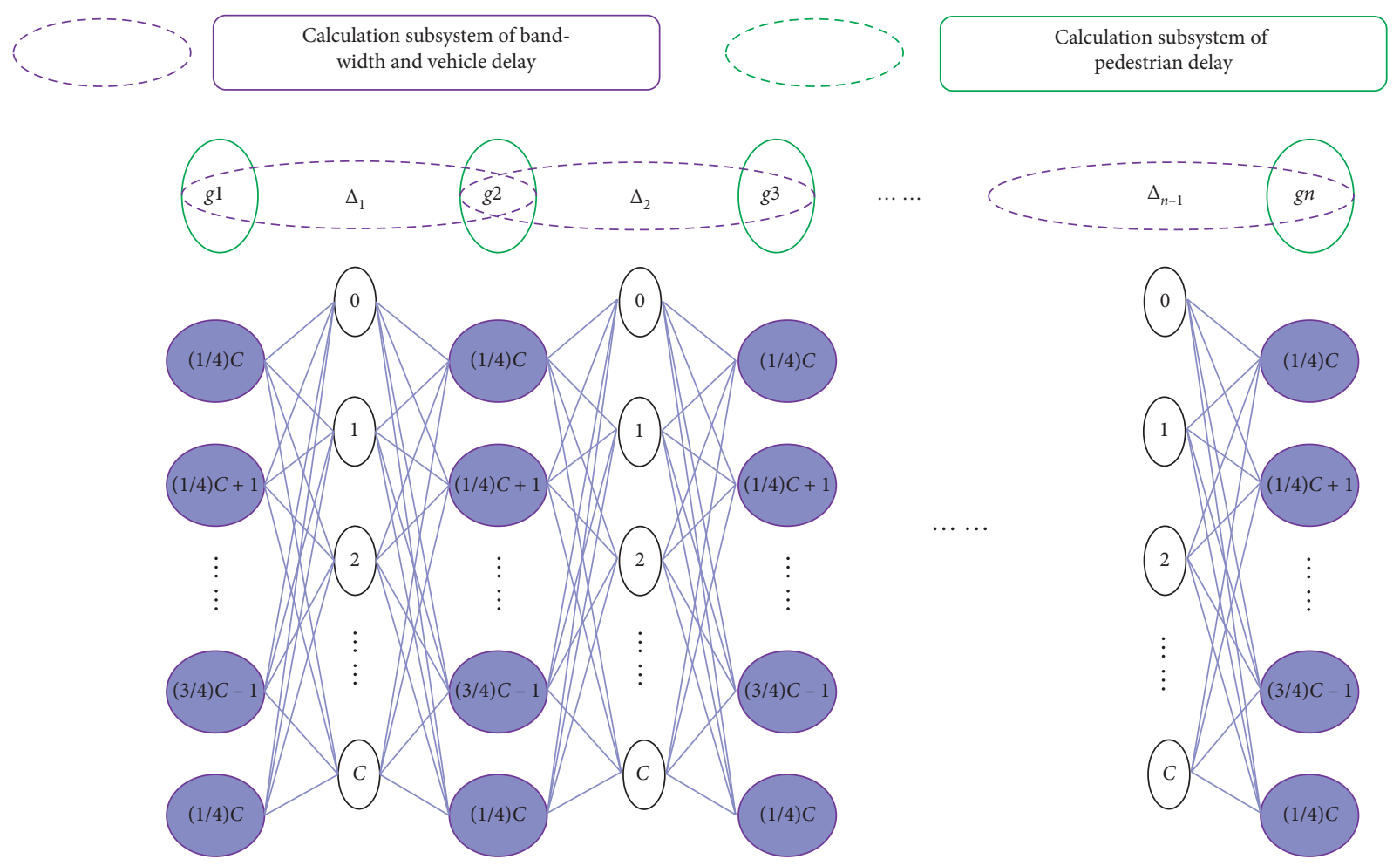

FIGURE 8: Subsystem of calculation in each layer.

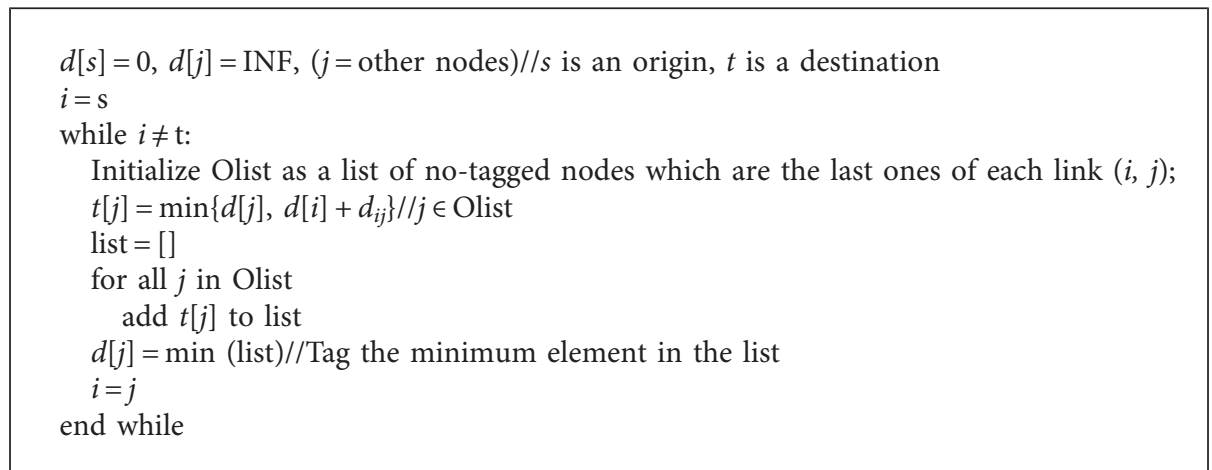

Algorithm 1: Pseudocode of the algorithm.

shopping, and other functions. There are Southeast University Sipailou Campus, Huahai 3C Plaza, Sipai Building Business Center and Presidential Palace and major learning, shopping, and office spaces such as libraries. Therefore, the traffic design of this main road is not only related to the efficiency of the entire urban transportation system, but also closely related to the work and life of the surrounding residents. It has important practical significance.

In addition, there are many places for students to study and conduct on this main road, including Southeast University and Chengxian Street Primary School. The walking demand is very large, which is in line with the background of pedestrians crossing the street. Furthermore, many intersections, such as Shipopo-Taiping North Road, Wendui Bridge-Taiping North Road, and Sipai
Building-Taiping North Road are T-shaped intersections, and Beijing East Road-Taiping North Road is only eastbound. In addition, some north-south roads are forbidden to turn left. All the conditions are similar to our assumption of coordinated control, that is, "The road between intersections does not contain a large number of vehicles that are transferred in or out" is similar. Therefore, this study selects this main road as the simulation object.

We collected corresponding data (flow, speed) by field investigation. Then according to the aforementioned algorithm, we can finally get the results of traffic signal coordinated control in Table 3 . Here we should note that the cycle length among the eight intersections is identical, that is, $120 \mathrm{~s}$, which is one of the assumptions in this study. Then we 


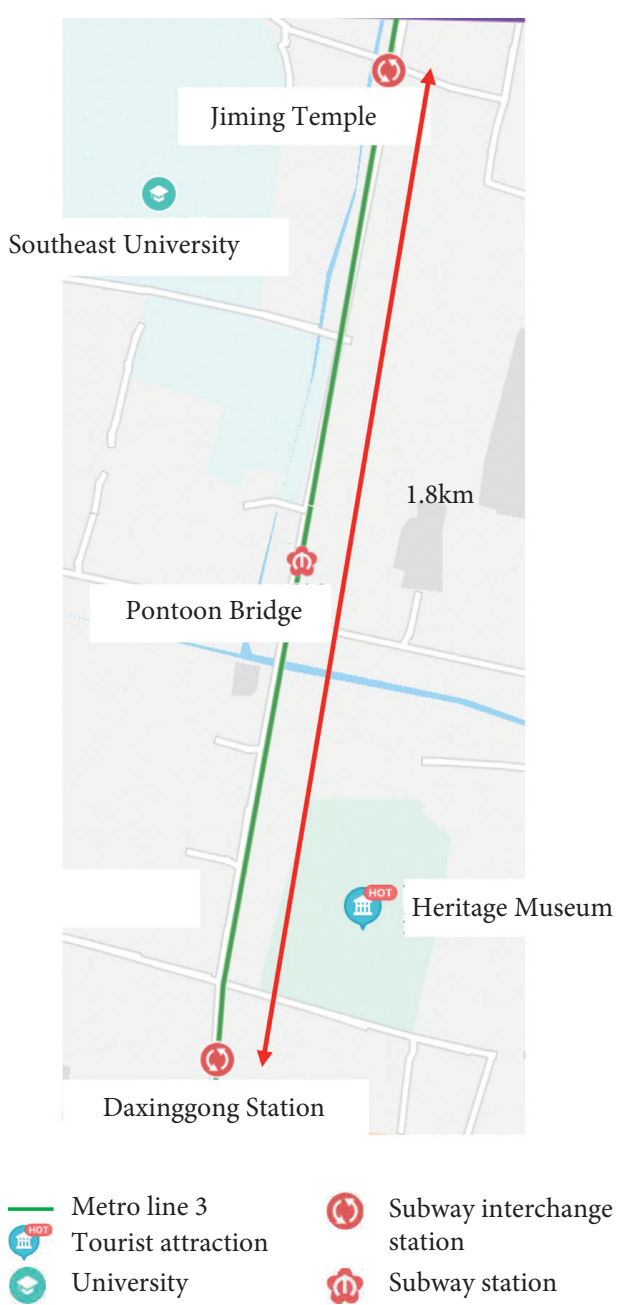

Figure 9: Artery road to be analyzed.

put the results in the microsimulator Vissim to test if the algorithm and models are reasonable.

Then we get final simulation results listed in Table 4 . We get classification of vehicle and pedestrian service levels to evaluate the traffic situation.

It can be seen that in addition to Zhongshan RoadTaiping North Road and Zhujiang Road-Taiping North Road, the delays of other coordinated intersections have been improved, and some service levels have even improved by one level. The average queue length is 4 to 8 vehicles in accordance with the average headway distance of $6 \mathrm{~m}$, which is acceptable. However, motor vehicle delays at the two complex intersections increased after the improvement, with an average queue of more than 15 vehicles. In terms of pedestrians, the average delay of the 8 intersections is within $30 \mathrm{~s}$, the service level was more in the $\mathrm{B}$ and $\mathrm{C}$ sections, and the effect on pedestrian control reached expectations.

In short, although the delay of the two separate intersections increased slightly, the control effect of the motor vehicles and pedestrians on the whole road was improved due to the formation of green waves, which basically achieves the purpose of the project, as well as motor vehicle progression band model, pedestrian delay model, and the rationality of the algorithm. To further improve the result, we will analysis and connect findings between branch-and-bound search [39] and algorithm in this paper.

\section{Conclusion and Discussions}

In this paper, a progression band model is established for the progression band and delay generated by the motor vehicle passing through adjacent intersections. On the basis of the existing model, the conditions are further refined, so that the model covers different intersection cycle length and green time, and the calculation formulas of progression bandwidth and delay under different conditions are given. In order to obtain a more accurate model for calculating pedestrian crossing delays, different models and formulas established in several documents were integrated, and their advantages and disadvantages and applicable conditions are analyzed. Furthermore, three kinds of motor vehicle flow speed were established with Vissim. In this case, different pedestrian flows are set from small to large, and the simulation delay is compared with the calculation delay. Finally, we get the 
TABLE 3: Data of intersection and optimized results.

\begin{tabular}{|c|c|c|c|c|c|c|c|}
\hline \multirow{2}{*}{ Intersection } & & \multirow[b]{2}{*}{ Flow (pcu/h) } & \multirow[b]{2}{*}{ Left-turn ratio } & \multirow{2}{*}{$\begin{array}{l}\text { Right- } \\
\text { turn ratio }\end{array}$} & \multirow[b]{2}{*}{ Pedestrian flow (ped/h) } & \multicolumn{2}{|c|}{ Optimized } \\
\hline & & & & & & $\begin{array}{l}\text { Green time } \\
(\mathrm{N}-\mathrm{S})(\mathrm{s})\end{array}$ & Offset (s) \\
\hline \multirow{4}{*}{ Beijing East Road-Taiping North Road } & $\mathrm{N}$ & 420 & 0.21 & 0.29 & 1352 & \multirow{4}{*}{63} & \\
\hline & S & 230 & 0.7 & 0.3 & 537 & & \\
\hline & $\mathrm{W}$ & 746 & 0 & 0.24 & 339 & & \\
\hline & $\mathrm{E}$ & - & - & - & 134 & & 35 \\
\hline \multirow{4}{*}{ Sipailou Road-Taiping North Road } & $\mathrm{N}$ & 583 & 0 & 0.18 & 147 & \multirow{4}{*}{85} & Jנ \\
\hline & $\mathrm{S}$ & 537 & 0.32 & 0 & 209 & & \\
\hline & $\mathrm{W}$ & 285 & 0.36 & 0.64 & 614 & & \\
\hline & $\mathrm{E}$ & - & - & - & - & & 104 \\
\hline \multirow{4}{*}{ Wendui Bridge-Taiping North Road } & $\mathrm{N}$ & 412 & 0 & 0 & 1462 & \multirow{4}{*}{83} & 104 \\
\hline & S & 564 & 0 & 0 & 253 & & \\
\hline & $\mathrm{W}$ & 103 & 0.33 & 0.67 & 638 & & \\
\hline & $\mathrm{E}$ & - & - & - & - & & 21 \\
\hline \multirow{5}{*}{ Zhujiang Road-Taiping North Road } & $\mathrm{N}$ & 692 & 0.32 & 0.06 & 1892 & \multirow{5}{*}{64} & 21 \\
\hline & $\mathrm{S}$ & 432 & 0.12 & 0.35 & 1379 & & \\
\hline & $\mathrm{W}$ & 772 & 0.10 & 0.16 & 960 & & \\
\hline & $\mathrm{E}$ & 750 & 0.27 & 0.05 & 1053 & & 3 \\
\hline & $\mathrm{N}$ & 1324 & 0.13 & 0.21 & 590 & & 3 \\
\hline \multirow{3}{*}{ Changjiang Back Street-Taiping North Road } & $\mathrm{S}$ & 1209 & 0.04 & 0.09 & 816 & \multirow{3}{*}{65} & \\
\hline & W & 301 & 0.35 & 0.29 & 1283 & & \\
\hline & $\mathrm{E}$ & 180 & 0.43 & 0.26 & 1720 & & 84 \\
\hline \multirow{5}{*}{ Shi Po Po-Taiping North Road } & $\mathrm{N}$ & 1190 & 0.24 & 0.06 & 24 & \multirow{5}{*}{58} & 84 \\
\hline & S & 891 & 0.32 & 0.16 & 528 & & \\
\hline & $\mathrm{W}$ & 289 & 0.14 & 0.33 & 730 & & \\
\hline & $\mathrm{E}$ & - & - & - & - & & 33 \\
\hline & $\mathrm{N}$ & 932 & 0.13 & 0.19 & 1625 & & \\
\hline \multirow{3}{*}{ Changjiang Road-Taiping North Road } & S & 1209 & 0.19 & 0.20 & 1793 & \multirow{4}{*}{61} & \\
\hline & $\mathrm{W}$ & 509 & 0.10 & 0.21 & 2019 & & \\
\hline & E & 734 & 0.32 & 0.11 & 1831 & & 13 \\
\hline \multirow{4}{*}{ Zhongshan East Road-Taiping North Road } & $\mathrm{N}$ & 1723 & 0.12 & 0.32 & 2032 & & 10 \\
\hline & S & 1439 & 0.23 & 0.17 & 1208 & \multirow{3}{*}{66} & \\
\hline & $\mathrm{W}$ & 1887 & 0.11 & 0.32 & 1902 & & \\
\hline & E & 2031 & 0.22 & 0.16 & 2319 & & \\
\hline
\end{tabular}

TABle 4: Simulation results compared with actual situation.

\begin{tabular}{|c|c|c|c|c|c|c|c|}
\hline \multirow[b]{2}{*}{ Intersection } & \multicolumn{3}{|c|}{ Before improvement } & \multicolumn{3}{|c|}{ After improvement } & \multirow[b]{2}{*}{$\begin{array}{c}\text { Ped service } \\
\text { level }\end{array}$} \\
\hline & Delay(s) & $\begin{array}{c}\text { Service } \\
\text { level }\end{array}$ & $\begin{array}{l}\text { Vehicle } \\
\text { delay(s) }\end{array}$ & $\begin{array}{c}\text { Queue } \\
\text { length }(\mathrm{m})\end{array}$ & $\begin{array}{c}\text { Service } \\
\text { level }\end{array}$ & $\begin{array}{c}\text { Ped } \\
\text { delay }(\mathrm{s})\end{array}$ & \\
\hline $\begin{array}{l}\text { Beijing East Road-Taiping North } \\
\text { Road }\end{array}$ & 38.74 & II & 22.56 & 30.34 & I & 13.24 & $\mathrm{~B}$ \\
\hline Sipailou Road-Taiping North Road & 45.92 & II & 37.34 & 41.43 & II & 22.56 & $\mathrm{C}$ \\
\hline Wendui Bridge-Taiping North Road & 58.31 & III & 34.02 & 55.28 & II & 31.34 & $\mathrm{D}$ \\
\hline Zhujiang Road-Taiping North Road & 79.10 & IV & 88.01 & 93.10 & IV & 28.79 & $\mathrm{C}$ \\
\hline $\begin{array}{l}\text { Changjiang Back street-Taiping } \\
\text { North Road }\end{array}$ & 44.73 & II & 31.83 & 28.49 & I & 25.14 & $\mathrm{C}$ \\
\hline Shi Po Po-Taiping North Road & 22.19 & I & 20.57 & 22.76 & I & 15.63 & $\mathrm{~B}$ \\
\hline $\begin{array}{l}\text { Changjiang Road-Taiping North } \\
\text { Road }\end{array}$ & 58.49 & III & 44.92 & 47.94 & II & 18.35 & B \\
\hline $\begin{array}{l}\text { Zhongshan East Road-Taiping North } \\
\text { Road }\end{array}$ & 88.31 & IV & 100.39 & 92.46 & IV & 30.79 & $\mathrm{D}$ \\
\hline
\end{tabular}

model and calculation formula with the best fitting degree under different conditions.

In order to fuse the above two models, a bilevel programming problem is established. Then, we analyze the characteristic of this study to simplify the objective function and use the shortest path searching to get the optimization results. In order to verify the rationality of the algorithm and the corresponding models, we find artery roads in line with the background of the project, conduct research and analysis, and obtain relevant data. After integration, on the one hand, the numerical classification will be carried into the algorithm to obtain the results of coordinated intersection, that is, cycle length, green time and offset, and the progression bandwidth based on the above models; on the other hand, the results are input into Vissim for simulation, in an environment with certain behavioral assumptions. The operation is carried out 


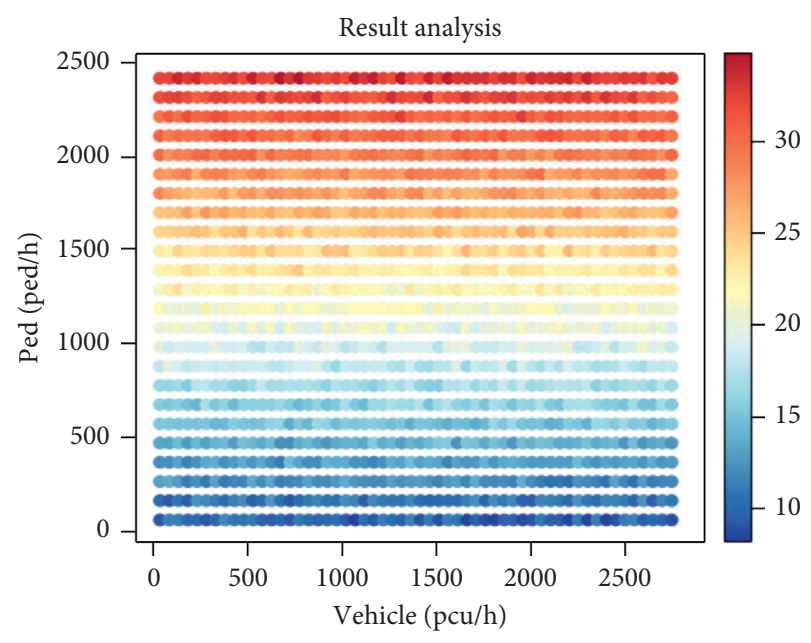

FIgURE 10: Scatter plot of pedestrian delay with two forms of flow.

TABLE 5: Clustering center of vehicle flow and pedestrian delay.

\begin{tabular}{lccc}
\hline & Cluster center & & \\
& & Result & \\
& 1 & 2 & 3 \\
\hline Vehicle flow & 600 & 2375 & 1450 \\
Pedestrian delay & 22 & 21 & 21 \\
\hline
\end{tabular}

to obtain the effect and evaluation index after the improvement under the actual situation. Finally, the service level analysis is carried out, and the certain rationality of the project model and algorithm can be guaranteed.

The biggest contribution of this paper is to put forward models of progression bandwidth and pedestrian delay and establish a correlation algorithm with intersections as subsystems to solve the corresponding signal coordination parameters.

Here is the future direction of research in my mind. In the establishment of the progression bandwidth model, it is assumed that the upstream vehicles are input with continuous and uniform green wave velocity in the green time. When multiple intersections are considered simultaneously, the situation becomes more and more complicated. For a driver there are two choices at the intersection, waiting or passing. Therefore, for a main road with $n$ intersections, there are $2^{n-1}$ possibilities for a car through the main road. In this paper, only the progression band of two adjacent intersections is simply analyzed, which is deviated from the actual situation. However, direct analysis is very complicated. Therefore, in the progression band model, how to efficiently analyze the green wave between multiple intersections becomes a research direction that needs in-depth study.

\section{Appendix}

\section{Cluster Analysis of the Relationship among Variables}

To get the dimensionality reduction and better know the relationship between pedestrian flow and two forms of flow,
TABLE 6: Clustering variance of vehicle flow and pedestrian delay.

\begin{tabular}{lcccccc}
\hline \multicolumn{7}{c}{ ANOVA } \\
& Cluster & Error & & \\
& $R^{2}$ & $\mathrm{~d} f$ & $R^{2}$ & $\mathrm{~d} f$ & $F$ & Sig. \\
\hline Vehicle flow & $3.902 \mathrm{E} 8$ & 2 & 72684.564 & 1341 & 5367.921 & 0.000 \\
Pedestrian delay & 0.916 & 2 & 48.236 & 1341 & 0.019 & 0.981 \\
\hline
\end{tabular}

we use K-means clustering. Firstly, $k$ points of clustering number are randomly selected as the starting center, then the remaining points and the points closest to the starting center are classified into one category, and the center of gravity of each category is calculated, then the center of gravity is used as the new center to continue iteration until the convergence conditions are met. In addition, this method needs a given number of clusters and is sensitive to abnormal data and data noise.

System clustering regards each object as an independent class, merges the two closest samples to form a new point, and stops when all the data are combined into a cluster or reaches some convergence rule. We simulate the relationship in Vissim and get the results in Figure 10.

From the figure, we can preliminarily find that the horizontal variation of delay is not obvious; that is, the relationship between pedestrian delay and vehicle flow is not significant. However, the vertical variation is obvious, indicating that the relationship between pedestrian delay and pedestrian flow is significant. In order to better explain the relationship between them, we will use cluster analysis to explain. In addition, according to the figure, we believe that clustering is carried out according to three categories.

Table 5 shows the clustering center of vehicle flow and pedestrian delay. Table 6 shows clustering variance of vehicle flow and pedestrian delay. Therefore, we can get the result that $q=600 \mathrm{pcu} / \mathrm{h}$ when the motor flow is small; $q=1500 \mathrm{pcu} / \mathrm{h}$ when the motor flow is moderate; $q=2400 \mathrm{pcu} / \mathrm{h}$ when the motor flow is large.

\section{Data Availability}

The traffic flow data used to support the findings of this study are available from the corresponding author upon request.

\section{Conflicts of Interest}

The authors declare that they have no conflicts of interest.

\section{Acknowledgments}

The study described in this paper was supported by the National Key Research and Development Program of China (No. 2018YFB1600900), the National Nature Science Foundation of China (No. 71971056, No. 51608115), the Six Talent Peaks Project in Jiangsu Province XNYQC-003, and the Open Project of the Key Laboratory of Advanced Urban Public Transportation Science, Ministry of Transport, PRC. This research was also jointly funded by research grants from the Research Grants Council of the Hong Kong Special 
Administrative Region (Project No. PolyU 15212217) and the Hong Kong Scholars Program (Project No. G-YZ1R).

\section{References}

[1] D. Li, T. Miwa, and T. Morikawa, "Considering en-route choices in utility-based route choice modelling," Networks and Spatial Economics, vol. 14, no. 3-4, pp. 581-604, 2014.

[2] D. Li, T. Miwa, T. Morikawa, and P. Liu, "Incorporating observed and unobserved heterogeneity in route choice analysis with sampled choice sets," Transportation Research Part C: Emerging Technologies, vol. 67, pp. 31-46, 2016.

[3] L. Cheng, X. Chen, S. Yang, Z. Cao, J. De Vos, and F. Witlox, "Active travel for active ageing in China: the role of built environment," Journal of Transport Geography, vol. 76, pp. 142-152, 2019.

[4] P. Liu, J. Wu, H. Zhou, J. Bao, and Z. Yang, "Estimating queue length for contraflow left-turn lane design at signalized intersections," Journal of Transportation Engineering, Part A: Systems, vol. 145, no. 6, pp. 1-9, 2019.

[5] Y. Pan, S. Chen, F. Qiao, S. V. Ukkusuri, and K. Tang, "Estimation of real-driving emissions for buses fueled with liquefied natural gas based on gradient boosted regression trees," Science of the Total Environment, vol. 660, pp. 741-750, 2019.

[6] Q. Cheng, S. Wang, Z. Liu, and Y. Yuan, "Surrogate-based simulation optimization approach for day-to-day dynamics model calibration with real data," Transportation Research Part C: Emerging Technologies, vol. 105, pp. 422-438, 2019.

[7] D. Li, T. Miwa, C. Xu, and Z. Li, "Non-linear fixed and multilevel random effects of origin-destination specific attributes on route choice behaviour," IET Intelligent Transport Systems, vol. 13, no. 4, pp. 654-660, 2019.

[8] D. Li, T. Miwa, and T. Morikawa, "Modeling time-of-day car use behavior: a Bayesian network approach," Transportation Research Part D: Transport and Environment, vol. 47, pp. 54-66, 2016.

[9] Y. Zhang, Y. Jiang, W. Rui, and R. G. Thompson, “Analyzing truck fleets' acceptance of alternative fuel freight vehicles in China," Renewable Energy, vol. 134, pp. 1148-1155, 2019.

[10] L. Li, J. Zhang, Y. Wang, and B. Ran, "Missing value imputation for traffic-related time series data based on a multiview learning method," IEEE Transactions on Intelligent Transportation Systems, vol. 20, no. 8, pp. 2933-2943, 2018.

[11] Y. Yuan, M. Yang, J. Wu, S. Rasouli, and D. Lei, “Assessing bus transit service from the perspective of elderly passengers in Harbin, China," International Journal of Sustainable Transportation, vol. 13, no. 10, pp. 761-776, 2019.

[12] J. T. Morgan and J. D. C. Little, "Synchronizing traffic signals for maximal bandwidth," Operations Research, vol. 12, no. 6, pp. 896-912, 1964.

[13] N. H. Gartner, S. F. Assman, F. Lasaga, and D. L. Hous, "MULTIBAND: a variable bandwidth arterial progression scheme," Transportation Research Record, vol. 1287, pp. 212-222, 1990.

[14] N. H. Gartner, S. F. Assman, F. Lasaga, and D. L. Hou, "A multi-band approach to arterial traffic signal optimization," Transportation Research Part B: Methodological, vol. 25, no. 1, pp. 55-74, 1991.

[15] R. Fernandez, E. Valenzuela, F. Casanello, and J. Carola, "Evolution of the TRANSYT model in a developing country," Transportation Research Part A: Policy and Practice, vol. 40, no. 5, pp. 386-398, 2005.
[16] S. Yagar and B. Han, "A procedure for real-time signal control that considers priority," Transportation Research Part B: Methodological, vol. 28, no. 4, pp. 315-331, 1994.

[17] M. Vasudevan, "Robust optimization model for bus priority under arterial progression," Ph. D. thesis, University of Maryland, College Park, MD, USA, 2005.

[18] P. Barford, J. Kline, D. Plonka, and A. Ron, "A signal analysis of network traffic anomalies," in Proceedings of ACM SIGCOMM Internet Measurement Workshop, Marseille, France, November 2002.

[19] A. L. C. Bazzan, "A distributed approach for coordination of traffic signal agents," Autonomous Agents and Multi-Agent Systems, vol. 10, no. 1, pp. 131-164, 2005.

[20] H. K. J. Lo, "A cell-based traffic control formulation: strategies and benefits of dynamic timing plans," Transportation Science, vol. 35, no. 2, pp. 148-164, 2001.

[21] M. Smith and T. van Vuren, "Traffic equilibrium with responsive traffic control," Transportation Science, vol. 27, no. 2, pp. 118-132, 1993.

[22] D. Bretherton, G. Bowen, and K. Wood, "Effective urban traffic management and control: SCOOT Version 4.4," in Proceedings of the European Transport Conference (CD-ROM), pp. 1-14, Cambridge, UK, 2002.

[23] C.-W. Tan, S. Park, H. Liu, Q. Xu, and P. Lau, "Prediction of transit vehicle arrival time for signal priority control: algorithm and performance," IEEE Transactions on Intelligent Transportation Systems, vol. 9, no. 4, pp. 688-696, 2008.

[24] Q. Cheng, Z. Liu, and W. Y. Szeto, "A cell-based dynamic congestion pricing scheme considering travel distance and time delay," Transportmetrica B: Transport Dynamics, vol. 7, no. 1, pp. 1286-1304, 2019.

[25] Y. Liu, Z. Liu, and R. Jia, "DeepPF: a deep learning based architecture for metro passenger flow prediction," Transportation Research Part C: Emerging Technologies, vol. 101, pp. 18-34, 2019.

[26] C. Wang, Z. Ye, E. Chen, M. Xu, and W. Wang, "Diffusion approximation for exploring the correlation between failure rate and bus-stop operation," Transportmetrica A: Transport Science, vol. 15, no. 2, pp. 1306-1320, 2019.

[27] C. Wang, C. Xu, J. Xia, Z. Qian, and L. Lu, “A combined use of microscopic traffic simulation and extreme value methods for traffic safety evaluation," Transportation Research Part C: Emerging Technologies, vol. 90, pp. 281-291, 2018.

[28] C. Xu, Y. Wang, P. Liu, W. Wang, and J. Bao, "Quantitative risk assessment of freeway crash casualty using high-resolution traffic data," Reliability Engineering \& System Safety, vol. 169, pp. 299-311, 2018.

[29] M. Du and L. Cheng, "Better understanding the characteristics and influential factors of different travel patterns in freefloating bike sharing: evidence from Nanjing, China," Sustainability, vol. 10, no. 4, p. 1244, 2018.

[30] Y. Guo, Z. Li, Y. Wu, and C. Xu, "Evaluating factors affecting electric bike users' registration of license plate in China using Bayesian approach," Transportation Research Part F: Traffic Psychology and Behaviour, vol. 59, pp. 212-221, 2018.

[31] Y. Ji, Y. Fan, A. Ermagun, X. Cao, W. Wang, and K. Das, "Public bicycle as a feeder mode to rail transit in China: the role of gender, age, income, trip purpose, and bicycle theft experience," International Journal of Sustainable Transportation, vol. 11, no. 4, pp. 308-317, 2017.

[32] H. Zhong-He, Z. Xing-Yuan, and L. Ying-Hong, "Dynamic programming approach for multi-bandwidth offset optimization," in Proceedings of the 28th Chinese Control Decision Conference CCDC, Hefei, China, May 2016. 
[33] R. Braun and M. Roddin, Quantifying the Benefits of Separating Pedestrians and Vehicles, Transportation Research Board, National Research Council, Washington, DC, USA, 1978.

[34] L. Qingfeng, Z. Wang, J. Yang, and J. Wang, "Pedestrian delay estimation at signalized intersections in developing cities," Transportation Research Part A: Policy and Practice, vol. 39, no. 1, pp. 61-73, 2005.

[35] T. Wang, J. Zhao, and C. Li, "Pedestrian delay model for continuous flow intersections under three design patterns," Mathematical Problems in Engineering, vol. 9, pp. 1-12, 2019.

[36] S. Marisamynathan and P. Vedagiri, "Modeling pedestrian delay at signalized intersections under mixed traffic conditions," Procedia, Social and Behavioral Sciences, vol. 104, pp. 708-717, 2013.

[37] A. Hajbabaie and R. F. R. Benekohal, "Traffic signal timing optimization," Transportation Research Record: Journal of the Transportation Research Board, vol. 2355, no. 1, pp. 10-19, 2013.

[38] R. Peñabaena-Niebles, V. Cantillo, J. L. Moura, and A. Ibeas, "Design and evaluation of a mathematical optimization model for traffic signal plan transition based on social cost function," Journal of Advanced Transportation, vol. 2017, Article ID 1943846, 12 pages, 2017.

[39] P. Li and X. Zhou, "Recasting and optimizing intersection automation as a connected-and-automated-vehicle (CAV) scheduling problem: a sequential branch-and-bound search approach in phase-time-traffic hypernetwork," Transportation Research Part B: Methodological, vol. 105, pp. 479506, 2017. 